\title{
MANIN PAIRS AND TOPOLOGICAL FIELD THEORY
}

\author{
E. GETZLER
}

The Lie algebra of first order differential operators on the circle, sometimes known as the Atiyah algebra, has a central extension parametrized by a real number $d$, the chiral algebra spanned by the stress-energy tensor $\mathrm{T}(z)$ and current $\mathrm{J}(z)$, with singular operator products

$$
\begin{aligned}
\mathrm{T}(z) \cdot \mathrm{T}(w) & \sim \frac{\frac{3}{2} d}{(z-w)^{4}}+\frac{2 \mathrm{~T}(w)}{(z-w)^{2}}+\frac{\partial \mathrm{T}(w)}{z-w}, \\
\mathrm{~T}(z) \cdot \mathrm{J}(w) & \sim \frac{\mathrm{J}(w)}{(z-w)^{2}}+\frac{\partial \mathrm{J}(w)}{z-w}, \\
\mathrm{~J}(z) \cdot \mathrm{J}(w) & \sim \frac{d}{(z-w)^{2}} .
\end{aligned}
$$

We say that a field $A(z)$ has weight $(a, q)$ if it has conformal dimension $a$ and charge $q$ :

$$
\begin{aligned}
& \mathrm{T}(z) \cdot A(w) \sim \frac{a A(w)}{(z-w)^{2}}+\frac{\partial A(w)}{z-w}, \\
& \mathrm{~J}(z) \cdot A(w) \sim \frac{q A(w)}{z-w} .
\end{aligned}
$$

Kazama describes in [14] a chiral algebra spanned by the fields $\mathrm{T}(z)$ and $\mathrm{J}(z)$, together with fermionic fields $\mathrm{G}^{+}(z), \mathrm{G}^{-}(z)$ and $\Phi(z)$ of weight $\left(\frac{3}{2}, 1\right),\left(\frac{3}{2},-1\right)$ and $\left(\frac{3}{2},-3\right)$ and a bosonic field $\mathrm{F}(z)$ of weight $(2,-2)$. These fields have singular operator products

$$
\begin{aligned}
\mathrm{G}^{+}(z) \cdot \mathrm{G}^{-}(w) & \sim \frac{d}{(z-w)^{3}}+\frac{\mathrm{J}(w)}{(z-w)^{2}}+\frac{\mathrm{T}(w)+\frac{1}{2} \partial \mathrm{J}(w)}{z-w}, \\
\mathrm{G}^{+}(z) \cdot \Phi(w) & \sim-\frac{1}{2} \mathrm{G}^{-}(z) \cdot \mathrm{G}^{-}(w) \sim \frac{F(w)}{z-w}, \\
\mathrm{G}^{+}(z) \cdot \mathrm{G}^{+}(w) & \sim \mathrm{G}^{-}(z) \cdot \Phi(w) \sim \mathrm{G}^{+}(z) \cdot F(w) \sim 0 \\
\mathrm{G}^{-}(z) \cdot F(w) & \sim \frac{3 \Phi(w)}{(z-w)^{2}}+\frac{\partial \Phi(w)}{z-w} .
\end{aligned}
$$

With $\Phi(z)$ and $F(z)$ set to zero, this chiral algebra becomes the $N=2$ superconformal algebra: for this reason, we will call it the $N=1 \frac{1}{2}$ superconformal algebra.

In this paper, we construct actions of the $N=1 \frac{1}{2}$ superconformal algebra, with as special cases the Kazama-Suzuki model, the $G / G$ model, and several deformations of

The author is partially supported by a fellowship of the Sloan Foundation, and a research grant of the NSF. 
these models. (The $G / G$ model has also been considered by Isidro-Ramallo [11].) The data from which we construct such an action are as follows:

(1) a finite-dimensional reductive Lie algebra $\mathfrak{g}$ with invariant inner product $(-,-)$;

(2) a polarization $\mathfrak{g}=\mathfrak{g}_{+} \oplus \mathfrak{g}_{-}$of $\mathfrak{g}$ (that is, a decomposition into a direct sum of transverse isotropic subspaces) such that $\mathfrak{g}_{+}$is a Lie subalgebra of $\mathfrak{g}$;

(3) a highest weight representation $\mathcal{E}$ of the affine Kac-Moody algebra $\widehat{\mathfrak{g}}$ with central extension represented by the operator product expansion

$$
X(z) \cdot Y(w) \sim \frac{(X, Y)-\frac{1}{2}\langle X, Y\rangle}{(z-w)^{2}}+\frac{[X, Y](w)}{z-w},
$$

where $\langle-,-\rangle$ is the Killing form of $\mathfrak{g}$;

(4) an element $\alpha \in \mathfrak{g}_{0}$, where $\mathfrak{g}_{0}$ is the intersection of the orthogonal complements of $\left[\mathfrak{g}_{+}, \mathfrak{g}_{+}\right]$and $\left[\mathfrak{g}_{-}, \mathfrak{g}_{-}\right]$.

The vector space underlying this representation of the $N=1 \frac{1}{2}$ algebra is the tensor product of the highest weight representation $\mathcal{E}$ and the fermionic Fock space associated to the polarized inner product space $\mathfrak{g}$. The central charge is

$$
d=\frac{1}{2} \operatorname{dim} \mathfrak{g}-(\rho, \rho)-(\alpha, \alpha)
$$

where $\rho=\left[x_{i}, x^{i}\right] \in \mathfrak{g}$; here $x_{i}$ and $x^{i}$ are dual bases of $\mathfrak{g}_{+}$and $\mathfrak{g}^{-}$, and we employ the summation convention for repeated indices.

The data (1) and (2) are known as a Manin pair, and have been introduced by Drinfeld in the study of quasi-Hopf algebras [5]. If in addition $\mathfrak{g}_{-}$is a Lie subalgebra of $\mathfrak{g}$, we say that $\left(\mathfrak{g}, \mathfrak{g}_{+}, \mathfrak{g}_{-}\right)$form a Manin triple; this notion lies at the foundations of the Leningrad school's approach to completely integrable systems Semenov-Tian-Shansky [20]. In this case, we obtain an action of the $N=2$ superconformal algebra. When $\alpha=0$, this action has been constructed by Spindel, Sevrin, Troust and van Proyen ([22], [21]); the model of Kazama-Suzuki [15] is a special case of their results. Note that in the case of a Manin triple, the element $\alpha \in \mathfrak{g}_{0}$ corresponds to a pair of characters $x_{ \pm} \mapsto\left(\alpha, x_{ \pm}\right)$of the two Lie algebras $\mathfrak{g}_{ \pm}$.

In Section 7, we will construct an equivalence between the $\operatorname{SL}(2) / \operatorname{SL}(2)$ model and the $\mathrm{SL}(2) / \mathrm{SO}(1)$ model with a non-zero value of $\alpha$ : the resulting equivalence of $N=1 \frac{1}{2}$ superconformal algebras generalizes the results of Aharony-Ganoor-et al. [1], Hu and Yu [10] and Frenkel [7], who showed that the cohomology of the two theories is isomorphic.

In an appendix, we explain the formulas which are used in the manipulation of operator products: while straightforward, their application leads to very complicated formulas, and the chances of making an error are greatly reduced by the availability of an excellent Mathematica package (Thielemans [23]). We also prove a result, found independently by Figueroa-O'Farill [6], which gives a small number of relations between fields $\mathrm{G}^{ \pm}$and $\Phi$ 
which must be verified in order for them to generate an action of the $N=1 \frac{1}{2}$ superconformal algebra.

This work was inspired by the article of Parkhomenko [17], who drew attention to the central role played in the work of Sevrin et al. [22] and [21] of Manin triples. We are also grateful to Bong Lian and Gregg Zuckerman for a number of interesting conversations.

\section{The $N=1 \frac{1}{2}$ Superconformal Algebra}

Recall the definition of the $N=1$ superconformal algebra: this is the chiral algebra spanned by the stress-energy tensor $\mathrm{T}(z)$ and a fermionic field $\mathrm{G}(z)$, and their derivatives, with operator products

$$
\begin{aligned}
& \mathrm{T}(z) \cdot \mathrm{T}(w) \sim \frac{\frac{3}{2} d}{(z-w)^{4}}+\frac{2 \mathrm{~T}(w)}{(z-w)^{2}}+\frac{\partial \mathrm{T}(w)}{z-w}, \\
& \mathrm{~T}(z) \cdot \mathrm{G}(w) \sim \frac{\frac{3}{2} \mathrm{G}(w)}{(z-w)^{2}}+\frac{\partial \mathrm{G}(w)}{z-w}, \\
& \mathrm{G}(z) \cdot \mathrm{G}(w) \sim \frac{2 d}{(z-w)^{3}}+\frac{2 \mathrm{~T}(w)}{z-w} .
\end{aligned}
$$

Here, $d$ is a real number called the central charge of the algebra. (This normalization of the central charge, related to the usual central charge $c$ by the formula $d=c / 3$, is more natural in the study of $N=2$ models.) This chiral algebra may be embedded in the $N=1 \frac{1}{2}$ superconformal algebra, by mapping $\mathrm{G}$ to $\mathrm{G}^{+}+\mathrm{G}^{-}+\Phi$ and $\mathrm{T}$ to $\mathrm{T}$.

Superfields allow us to express these operator products in a compact way. In the complex superspace with coordinates $Z=(z, \theta)$, introduce the differential operator

$$
\mathcal{D}=\frac{\partial}{\partial \theta}+\theta \frac{\partial}{\partial z}
$$

so that $\mathcal{D}^{2}=\partial$. If $n \in \mathbb{Z}, \varepsilon \in\{0,1\}$ and $W=(w, \xi)$, it is convenient to adopt the notations

$$
\begin{aligned}
\frac{\mathcal{A}(W)}{(Z-W)^{n}} & =\frac{\mathcal{A}(W)}{(z-w-\theta \xi)^{n}}=\left((z-w)^{-n}+n \theta \xi(z-w)^{-n-1}\right) \mathcal{A}(W), \\
\frac{\mathcal{A}(W)}{(Z-W)^{n-\frac{1}{2}}} & =\frac{2(\theta-\xi) \mathcal{A}(W)}{(z-w-\theta \xi)^{n}}=\frac{2(\theta-\xi) \mathcal{A}(W)}{(z-w)^{n}} .
\end{aligned}
$$

Introducing the superfield $\mathcal{G}(Z)=\mathrm{G}(z)+2 \theta \mathrm{T}(z)$, the defining relations of the $N=1$ superconformal algebra are contained in the singular operator product

$$
\mathcal{G}(Z) \cdot \mathcal{G}(W) \sim \frac{2 d}{(Z-W)^{3}}+\frac{\frac{3}{2} \mathcal{G}(W)}{(Z-W)^{3 / 2}}+\frac{\mathcal{D} \mathcal{G}(W)}{Z-W}+\frac{\partial \mathcal{G}(W)}{(Z-W)^{1 / 2}} .
$$

A superfield $\Omega$ is a primary of dimension $\Delta$ if

$$
\mathcal{G}(Z) \cdot \Omega(W) \sim \frac{\Delta \Omega(W)}{(Z-W)^{3 / 2}}+\frac{\mathcal{D} \Omega(W)}{Z-W}+\frac{\partial \Omega(W)}{(Z-W)^{1 / 2}} .
$$


The $N=1$ superconformal algebra may be realized on the Fock space of a scalar field $\phi(z)$, with singular operator product

$$
\phi(z) \cdot \phi(w) \sim-\log (z-w)
$$

tensored with the Fock space of a free fermionic field $\psi(z)$ with singular operator product

$$
\psi(z) \cdot \psi(w) \sim \frac{1}{z-w} .
$$

The superfield $\Psi(Z)=\psi(z)+i \theta \partial \phi(z)$ is a primary of dimension $1 / 2$, and

$$
\Psi(Z) \cdot \Psi(W) \sim \frac{1}{Z-W}
$$

The superfield $\mathcal{G}=\Psi \mathcal{D} \Psi$ realizes the $N=1$ superconformal algebra with central charge $d=1 / 2$, and the fields $\mathrm{T}$ and $\mathrm{G}$ are given by the formulas

$$
\begin{aligned}
& \mathrm{T}=-\frac{1}{2}(\partial \phi)^{2}-\frac{1}{2} \psi \partial \psi, \\
& \mathrm{G}=i \partial \phi \psi
\end{aligned}
$$

The following simple result is an analogue for the $N=1$ superconformal algebra of the well-known deformation $\mathrm{T}+\alpha \partial J$ of a stress-energy tensor by the derivative of a current.

Proposition 1.1. If $\mathcal{G}$ is a superfield realizing the $N=1$ superconformal algebra with central charge $d$, and $\Phi$ is a primary fermionic superfield of dimension $1 / 2$ such that

$$
\Psi(Z) \cdot \Psi(W) \sim \frac{1}{Z-W}
$$

then $\mathcal{G}+\alpha \partial \Psi$ realizes the $N=1$ superconformal algebra with central charge $d-\alpha^{2}$.

In the case of the free field realization, we obtain a deformation with central charge $d-\alpha^{2}$, given explicitly by the formulas

$$
\begin{aligned}
& \mathbf{T}=-\frac{1}{2}(\partial \phi)^{2}-\frac{1}{2} \psi \partial \psi+\frac{i}{2} \alpha \partial^{2} \phi \\
& \mathbf{G}=i \partial \phi \psi+\alpha \partial \psi
\end{aligned}
$$

The free-field realization of the $N=1$ superconformal algebra has a generalization, in which the place of the vector space $\mathbb{R}$ is taken by a reductive Lie algebra $\mathfrak{g}$ with invariant inner product $(-,-)$. Let $x_{i}$ be a basis of $\mathfrak{g}$, relative to which $\left[x_{i}, x_{j}\right]=C_{i j}^{k}$ and $h_{i j}=\left(x_{i}, x_{j}\right)$, with matrix inverse $h^{i j}$. Denote $h_{k l} C_{i j}^{l}$ by $C_{i j k}$ : it is antisymmetric in all three indices, reflecting the invariance of the inner product.

Let $\langle-,-\rangle$ be the Killing form of $\mathfrak{g}$, with coefficients

$$
g_{i j}=\left\langle x_{i}, x_{j}\right\rangle=C_{i k}^{l} C_{j l}^{k}
$$


Let $\mathfrak{g}$ be a simple Lie algebra with Cartan subalgebra $\mathfrak{h}^{*}$ and highest root $\theta$. Let $h$ be the dual Coxeter number of $\mathbf{k}$. We say that an inner product $(-,-)$ has level $k$ if

$$
\frac{(x, x)}{k+h}=\frac{\langle x, x\rangle}{2 h}
$$

The Killing form itself has level $h$.

Consider the chiral algebra generated by fields $J_{i}(z)$ satisfying the operator product expansions

$$
J_{i}(z) \cdot J_{j}(w)=\frac{h_{i j}-\frac{1}{2} g_{i j}}{(z-w)^{2}}+\frac{C_{i j}^{k} J_{k}(w)}{z-w} .
$$

These relations define a representation of the affine Kac-Moody algebra $\widehat{\mathfrak{g}}$, with central extension corresponding to the invariant inner product $(x, y)-\frac{1}{2}\langle x, y\rangle$ on $\mathfrak{g}$. Introduce free fermionic fields $a^{i}(z)$, with operator products

$$
a^{i}(z) \cdot a^{j}(w) \sim \frac{h^{i j}}{z-w} .
$$

The superfields $\mathcal{J}_{i}(Z)=h_{i j} a^{j}(z)+\theta\left(J_{i}-\frac{1}{2} C_{i j k} a^{j} a^{k}\right)$ realize the super Kac-Moody algebra associated to $\mathfrak{g}$ (Kac-Todorov [12]),

$$
\mathcal{J}_{i}(Z) \cdot \mathcal{J}_{j}(W) \sim \frac{h_{i j}}{Z-W}+\frac{\frac{1}{2} C_{i j}^{k} \mathcal{J}_{k}(w)}{(Z-W)^{1 / 2}} .
$$

The fields

$$
\begin{aligned}
& \mathrm{G}=J_{i} a^{i}-\frac{1}{6} C_{i j k} a^{i} a^{j} a^{k}, \\
& \mathbf{T}=\frac{1}{2}\left(h^{i j} J_{i} J_{j}+h_{i j} \partial a^{i} a^{j}\right),
\end{aligned}
$$

realize the $N=1$ superconformal algebra, with central charge $d=\frac{1}{2} \operatorname{dim} \mathfrak{g}-\frac{1}{6} h_{i j} g^{i j}$. When $\mathfrak{g}$ is abelian, the Killing form vanishes, and this model becomes the free-field realization of the $N=1$ superconformal chiral algebra.

The superfield $\mathcal{J}$ is a primary of dimension 1 ; it follows that if $\alpha$ is an element of $\mathfrak{g}$, we may deform $\mathcal{G}$ to $\mathcal{G}+(\alpha, \mathcal{D J})$, obtaining a realization of the $N=1$ superconformal algebra with central charge $d=\frac{1}{2} \operatorname{dim} \mathfrak{g}-\frac{1}{6} h_{i j} g^{i j}-(\alpha, \alpha)$.

The problem which we discuss in this paper is the construction of actions of the $N=1 \frac{1}{2}$ superconformal symmetry underlying this $N=1$ superconformal symmetry.

We may assemble the fields of the $N=1 \frac{1}{2}$ superconformal algebra into superfields by the following formulas:

$$
\begin{aligned}
& \mathcal{G}(Z)=\left(\mathrm{G}^{+}(z)+\mathrm{G}^{-}(z)+\Phi(z)\right)+2 \theta \mathrm{T}(z), \\
& \mathcal{J}(Z)=-\mathrm{J}(z)+\theta\left(\mathrm{G}^{+}(z)-\mathrm{G}^{-}(z)-3 \Phi(z)\right), \\
& \mathcal{F}(Z)=\Phi(z)+\theta \mathrm{F}(z)
\end{aligned}
$$


Then $\mathcal{G}$ generates an $N=1$ superconformal symmetry, $\mathcal{J}$ and $\mathcal{F}$ are bosonic and fermionic superfields of dimension 1 and $\frac{3}{2}$, and

$$
\begin{aligned}
\mathcal{J}(Z) \cdot \mathcal{J}(W) & \sim \frac{d}{(Z-W)^{2}}+\frac{\frac{1}{2} \mathcal{G}(W)+\mathcal{F}(W)}{(Z-W)^{1 / 2}}, \\
\mathcal{J}(Z) \cdot \mathcal{F}(W) & \sim \frac{3 \mathcal{F}(W)}{Z-W}+\frac{\frac{1}{2} \mathcal{D} \mathcal{F}(W)}{(Z-W)^{1 / 2}}, \\
\mathcal{F}(Z) \cdot \mathcal{F}(W) & \sim 0 .
\end{aligned}
$$

We may think of $\mathcal{F}$ as a superfield representing a curvature term.

When the curvature $\mathcal{F}$ equals zero and we have the $N=2$ superconformal algebra, there is an automorphism which sends $\mathcal{J}$ to $-\mathcal{J}$, and leaves $\mathcal{G}$ fixed. In terms of the component fields, this exchanges $\mathrm{G}^{+}$and $\mathrm{G}^{-}$, and sends $\mathrm{J}$ to $-\mathrm{J}$. There is no analogue of this automorphism when $\mathcal{F}$ is non-zero.

The following result, which has been found independently by Figueroa-O'Farill [6] when $\Phi=\mathrm{F}=0$, simplifies the verification of these operator products in concrete situations. The proof is contained in the Appendix, along with some background on vertex algebras used in its proof.

Proposition 1.2. Let $\mathrm{G}^{ \pm}(z)$ and $\Phi(z)$ be fermionic fields such that

$$
\begin{aligned}
\mathrm{G}^{+}(z) \cdot \mathrm{G}^{-}(w) & \sim \frac{d}{(z-w)^{3}}+\frac{\mathrm{J}(w)}{(z-w)^{2}}+\frac{T(w)+\frac{1}{2} \partial \mathrm{J}(w)}{z-w}, \\
\mathrm{G}^{+}(z) \cdot \Phi(w) & \sim-\frac{1}{2} \mathrm{G}^{-}(z) \cdot \mathrm{G}^{-}(w) \sim \frac{\mathrm{F}(w)}{z-w} \\
\mathrm{G}^{+}(z) \cdot \mathrm{G}^{+}(w) & \sim 0
\end{aligned}
$$

for a scalard and bosonic fields $\mathrm{J}(z), \mathrm{T}(z)$ and $\mathrm{F}(z)$, and

$$
\mathrm{J}(z) \cdot \mathrm{G}^{ \pm}(w) \sim \pm \frac{\mathrm{G}^{ \pm}(w)}{z-w}, \quad \mathrm{~J}(z) \cdot \Phi(w) \sim-\frac{3 \Phi(w)}{z-w} .
$$

Then these fields satisfy the operator products of the $N=1 \frac{1}{2}$ superconformal algebra, with central charge $d$.

The following corollary applies to the case when the curvature $\mathcal{F}$ vanishes.

Corollary 1.3. Let $\mathrm{G}^{ \pm}(z)$ be fermionic fields such that $\mathrm{G}^{ \pm}(z) \cdot \mathrm{G}^{ \pm}(w) \sim 0$,

$$
\mathrm{G}^{+}(z) \cdot \mathrm{G}^{-}(w) \sim \frac{d}{(z-w)^{3}}+\frac{\mathrm{J}(w)}{(z-w)^{2}}+\frac{T(w)+\frac{1}{2} \partial \mathrm{J}(w)}{z-w},
$$

for a scalar $d$ and bosonic fields $\mathrm{J}(z)$ and $\mathrm{T}(z)$, and

$$
\mathrm{J}(z) \cdot \mathrm{G}^{ \pm}(w) \sim \pm \frac{\mathrm{G}^{ \pm}(w)}{z-w} .
$$

Then these fields satisfy the operator products of the $N=2$ superconformal algebra. 
The following result provides an analogue of Proposition 1.1 for the $N=1 \frac{1}{2}$ superconformal algebra.

Proposition 1.4. Suppose $\Omega^{ \pm}(Z)$ are fermionic superfields such that

$$
\begin{aligned}
\mathcal{G}(Z) \cdot \Omega^{ \pm}(W) & \sim \frac{\frac{1}{2} \Omega^{ \pm}(W)}{(Z-W)^{3 / 2}}+\frac{\mathcal{D} \Omega^{ \pm}(W)}{Z-W}+\frac{\partial \Omega^{ \pm}(W)}{(Z-W)^{1 / 2}}, \\
\mp \mathcal{J}(Z) \cdot \Omega^{ \pm}(W) & \sim \frac{\Omega^{ \pm}(W)}{Z-W}+\frac{\frac{1}{2} \mathcal{D} \Omega^{ \pm}(W)}{(Z-W)^{1 / 2}}, \\
\Omega^{ \pm}(Z) \cdot \Omega^{ \pm}(W) & \sim \mathcal{F}(Z) \cdot \Omega^{ \pm}(W) \sim 0, \\
\Omega^{+}(Z) \cdot \Omega^{-}(W) & \sim \frac{d^{\prime}}{Z-W} .
\end{aligned}
$$

Then $\widetilde{\mathcal{G}}=\mathcal{G}+\partial \Omega^{+}+\partial \Omega^{-}, \widetilde{\mathcal{J}}=\mathcal{J}-\mathcal{D} \Omega^{+}+\mathcal{D} \Omega^{-}$and $\widetilde{\mathcal{F}}=\mathcal{F}$ define a realization of the $N=1 \frac{1}{2}$ superconformal algebra with central extension $d-2 d^{\prime}$.

Proof. Write $\Omega^{ \pm}(Z)=A^{ \pm}(z)+\theta B^{ \pm}(z)$. Then

$$
\begin{aligned}
& \mathrm{G}^{ \pm}(z) \cdot A^{ \pm}(w) \sim \frac{B^{ \pm}(w)}{z-w}, \\
& \mathrm{G}^{\mp}(z) \cdot A^{ \pm}(w) \sim \mathrm{G}^{ \pm}(z) \cdot B^{ \pm}(w) \sim 0 \\
& \mathrm{G}^{\mp}(z) \cdot B^{ \pm}(w) \sim \frac{A^{ \pm}(w)}{(z-w)^{2}}+\frac{\partial A^{ \pm}(w)}{z-w}, \\
& A^{+}(z) \cdot A^{-}(w) \sim \frac{d^{\prime}}{z-w}, \quad B^{+}(z) \cdot B^{-}(w) \sim \frac{d^{\prime}}{(z-w)^{2}},
\end{aligned}
$$

while $\Phi(z) \cdot A^{ \pm}(w) \sim A^{ \pm}(z) \cdot B^{ \pm}(w) \sim A^{ \pm}(z) \cdot B^{\mp}(w) \sim 0$. The deformed fields are given by the formulas

$$
\widetilde{\mathrm{T}}=\mathrm{T}+\frac{1}{2}\left(\partial B^{+}+\partial B^{-}\right), \quad \widetilde{\mathrm{G}}^{ \pm}=\mathrm{G}^{ \pm}+\partial A^{ \pm}, \quad \widetilde{\mathrm{J}}=\mathrm{J}+B^{+}-B^{-},
$$

while $\widetilde{\Phi}=\Phi$ and $\widetilde{F}=F$. The verification of the hypotheses of Proposition 1.2 is now straightforward.

\section{MANIN PAIRS}

The following definition is due to Drinfeld [5].

Definition 2.1. Let $\mathfrak{g}$ be a finite dimensional, reductive Lie algebra over $\mathbb{C}$, with invariant inner product $(-,-)$. A Manin pair $\left(\mathfrak{g}, \mathfrak{g}_{+}, \mathfrak{g}_{-}\right)$is a polarization $\mathfrak{g}=\mathfrak{g}_{+} \oplus \mathfrak{g}_{-}$of $\mathfrak{g}$ such that $\mathfrak{g}_{+}$is a Lie subalgebra of $\mathfrak{g}$; it is a Manin triple if in addition $\mathfrak{g}_{-}$is a Lie subalgebra of $\mathfrak{g}$.

In fact, Drinfeld does not include the complementary isotropic subspace $\mathfrak{g}_{-}$in the data defining a Manin pair: however, we find our more precise notion of a Manin pair to be convenient for the purposes of this paper. 
Given a Manin pair, define the subspace $\mathfrak{g}_{0} \subset \mathfrak{g}$ to be the intersection of the subspaces $\left[\mathfrak{g}_{+}, \mathfrak{g}_{+}\right]^{\perp}$ and $\left[\mathfrak{g}_{-}, \mathfrak{g}_{-}\right]^{\perp}$.

An example of a Manin pair is obtained by taking a reductive Lie algebra $\mathbf{k}$ with an invariant inner product $(-,-)$ : we let $\mathfrak{g}=\mathbf{k} \oplus \mathbf{k}$, with inner product

$$
\left(x_{1} \oplus y_{1}, x_{2} \oplus y_{2}\right)=\left(x_{1}, x_{2}\right)-\left(y_{1}, y_{2}\right) .
$$

Let $\mathfrak{g}_{ \pm}=\{x \oplus \pm x \mid x \in \mathbf{k}\}$ : it is clear that these subspaces are isotropic, and that $\mathfrak{g}_{+}$ is a Lie subalgebra (though not $\mathfrak{g}_{-}$, since $\left[\mathfrak{g}_{-}, \mathfrak{g}_{-}\right] \subset \mathfrak{g}_{+}$). This Manin pair is associated, by the construction of this paper, to the action of the $N=1 \frac{1}{2}$ superconformal algebra in the $G / G$ model.

Examples of Manin triples may be obtained by taking the following data:

(1) a simple Lie algebra $\mathbf{k}$, with Borel subalgebra $\mathbf{b}$;

(2) an invariant inner product $(-,-)$ on $\mathbf{k}$;

(3) a parabolic subalgebra $\mathbf{p} \supset \mathbf{b}$.

The parabolic subalgebra $\mathbf{p}$ has the Levi decomposition $\mathbf{p}=\mathbf{l} \oplus \mathbf{n}$, where $\mathbf{l}$ is reductive and $\mathbf{n}$ is nilpotent. Let $\mathfrak{g}$ be the Lie algebra $\mathfrak{g}=\mathbf{k} \oplus \mathbf{l}$, with inner product

$$
\left(x_{1} \oplus y_{1}, x_{2} \oplus y_{2}\right)=\left(x_{1}, x_{2}\right)-\left(y_{1}, y_{2}\right)
$$

The subalgebra

$$
\mathfrak{g}_{+}=\{x \oplus y \in \mathbf{k} \oplus \mathbf{l} \mid x-y \in \mathbf{n}\} \subset \mathfrak{g}
$$

is easily seen to be isotropic, and $\operatorname{dim} \mathfrak{g}_{+}=\frac{1}{2} \operatorname{dim} \mathfrak{g}$.

Let $\mathfrak{h}$ be the Cartan subalgebra of $\mathbf{k}$ determined by the Borel subalgebra $\mathbf{b}$; if $x \in \mathbf{k}$, denote by $x_{\mathfrak{h}}$ its projection onto $\mathfrak{h}$. Let $\mathbf{b}_{-}$be the Borel subalgebra opposite to $\mathbf{b}$. The subalgebra

$$
\mathfrak{g}_{-}=\left\{x \oplus y \in(\mathbf{k} \cap \mathbf{b}-) \oplus(\mathbf{l} \cap \mathbf{b}) \mid(x+y)_{\mathfrak{h}}=0\right\} \subset \mathfrak{g}
$$

is isotropic, and together with $\mathfrak{g}_{+}$forms a Manin triple.

The two extreme cases of this construction are of especial interest:

(1) If $\mathbf{p}=\mathbf{b}$ is itself a Borel subalgebra, then $\mathfrak{g}=\mathbf{k} \oplus \mathfrak{h}$ and

$$
\mathfrak{g}_{ \pm}=\left\{x \oplus h \in \mathbf{b}_{ \pm} \oplus \mathfrak{h} \mid x_{\mathfrak{h}}= \pm h\right\}
$$

This Manin triple corresponds to the Kazama-Suzuki model.

(2) If $\mathbf{p}=\mathbf{k}$ is all of $\mathbf{k}$, then $\mathfrak{g}=\mathbf{k} \oplus \mathbf{k}$ and

$$
\begin{aligned}
& \mathfrak{g}_{+}=\{x \oplus x \in \mathbf{k} \oplus \mathbf{k}\}, \\
& \mathfrak{g}_{-}=\left\{x \oplus y \in \mathbf{b}_{-} \oplus \mathbf{b}_{+} \mid x_{\mathfrak{h}}+y_{\mathfrak{h}}=0\right\} .
\end{aligned}
$$

This Manin triple corresponds to the $G / G$ model (and is also familiar as the Manin triple associated to the classical limit of the quantum group $U_{q} \mathbf{k}$ ). 
Denote by $\Delta \subset \mathfrak{h}^{*}$ the roots of $\mathbf{k}$, and by $\tilde{\Delta} \subset \Delta$ the roots of $\mathbf{l} \subset \mathbf{p}$. Let $\Pi \subset \Delta$ be the basis of the root system $\Delta$ determined by $\mathbf{b}$, and let $\Delta=\Delta_{+} \cup \Delta_{-}$be the associated decomposition of $\Delta$ into positive and negative roots. Then $\mathbf{p}$ has the decomposition into a sum of weight spaces of $\mathbf{k}$,

$$
\mathbf{p}=\sum_{\alpha \in \Delta_{+} \cup \tilde{\Delta}} \mathbf{k}_{\alpha}
$$

where for $\alpha \in \Delta, \mathbf{k}_{\alpha}=\{x \in \mathbf{k} \mid H x=\alpha(H) x$ for all $H \in \mathfrak{h}\}$.

To a root $\alpha \in \Delta \subset \mathfrak{h}^{*}$ is associated a coroot $\alpha^{\swarrow} \in \Delta^{\smile} \subset \mathfrak{h}$, such that the reflection $s_{\alpha}$ is given by the formula

$$
s_{\alpha} \beta=\beta-\alpha\left(\beta^{\curlyvee}\right)
$$

in particular, $\alpha\left(\alpha^{\sim}\right)=2$. For $\alpha \in \Delta_{+}$, let $x_{\alpha} \in \mathbf{k}_{\alpha}$ and $x_{-\alpha} \in \mathbf{k}_{-\alpha}$ be vectors such that

$$
\left[x_{\alpha}, x_{-\alpha}\right]=\alpha^{\llcorner}, \quad\left[\alpha^{\sim}, x_{ \pm \alpha}\right]= \pm 2 x_{\alpha} .
$$

It follows that

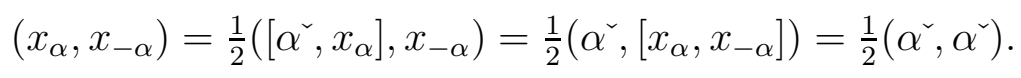

Given the Manin triple $\left(\mathfrak{g}=\mathbf{k} \oplus \mathbf{l}, \mathfrak{g}_{+}, \mathfrak{g}_{-}\right), \mathfrak{g}_{+}$has basis

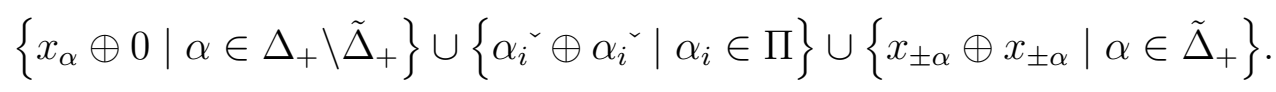

If $\left\{\alpha_{i} \mid 1 \leq i \leq r\right\}$ are the simple roots of $\mathbf{k}$, the fundamental weights $\left\{\omega_{i} \mid 1 \leq i \leq\right.$ $r\} \subset \mathfrak{h}^{*}$ are the dual basis of the basis $\left\{\alpha_{i}{ }_{i} \mid 1 \leq i \leq r\right\}$ of $\mathfrak{h}$. We may identify the weights $\omega_{i}$ with elements of $\mathfrak{h}$ by means of the restriction to $\mathfrak{h}$ of the inner product $(-,-)$. With these notations, the basis of $\mathfrak{g}_{-}$dual to the above basis of $\mathfrak{g}_{+}$is

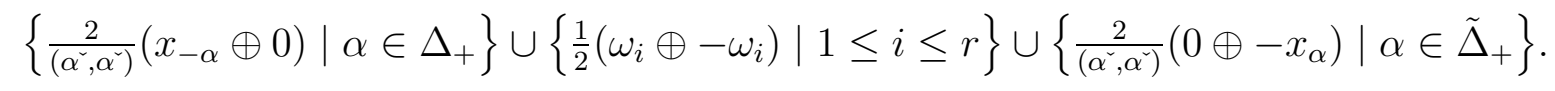

Thus, $\mathfrak{g}_{0}$ has the basis

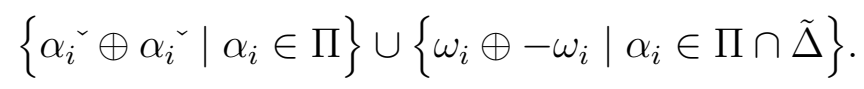

If $\left(\mathfrak{g}, \mathfrak{g}_{+}, \mathfrak{g}_{-}\right)$is a Manin triple, the inner product on $\mathfrak{g}$ induces an identification of $\mathfrak{g}_{-}$ with the dual $\mathfrak{g}_{+}^{*}$ of $\mathfrak{g}_{+}$, and vice versa. The choice of a basis $x_{i}$ of $\mathfrak{g}_{+}$determines a dual basis $x^{i}$ of $\mathfrak{g}_{-}$, such that the bracket of $\mathfrak{g}$ is given by the formulas

$$
\begin{aligned}
& {\left[x_{i}, x_{j}\right]=c_{i j}^{k} x_{k},} \\
& {\left[x_{i}, x^{j}\right]=f_{i}^{j k} x_{k}+c_{k i}^{j} x^{k},} \\
& {\left[x^{i}, x^{j}\right]=f_{k}^{i j} x^{k}-\phi^{i j k} x_{k} .}
\end{aligned}
$$


The coefficients $c_{i j}^{k}, f_{k}^{i j}$ and $\phi^{i j k}$ satisfy the identities

$$
\begin{aligned}
& c_{i j}^{k}=-c_{j i}^{k}, \quad f_{k}^{i j}=-f_{k}^{j i}, \quad \phi^{i j k}=-\phi^{j i k}=-\phi^{i k j}, \\
& c_{i j}^{m} c_{m k}^{l}+c_{j k}^{m} c_{m i}^{l}+c_{k i}^{m} c_{m j}^{l}=0, \\
& f_{m}^{i j} f_{l}^{m k}+f_{m}^{j k} f_{l}^{m i}+f_{m}^{k i} f_{l}^{m j}=c_{l m}^{i} \phi^{j k m}+c_{l m}^{j} \phi^{k i m}+c_{l m}^{k} \phi^{i j m}, \\
& c_{m k}^{i} f_{l}^{j m}-c_{m l}^{i} f_{k}^{j m}-c_{m k}^{j} f_{l}^{i m}+c_{m l}^{j} f_{k}^{i m}=c_{k l}^{m} f_{m}^{i j}, \\
& f_{m}^{k l} \phi^{i j m}+f_{m}^{i l} \phi^{j k m}+f_{m}^{j l} \phi^{k i m}=0 .
\end{aligned}
$$

Being a Manin triple is equivalent to the vanishing of the coefficients $\phi^{i j k}$.

Let us illustrate these formulas in the case of the Manin pair associated to $\mathbf{k} \oplus \mathbf{k}$. If we choose a basis $x_{i}$ of $\mathbf{k}$ with respect to which the inner product is given by the matrix $h_{i j}$, we obtain dual bases $x_{i} \oplus x_{i}$ and $\frac{1}{2} h^{i j}\left(x_{j} \oplus-x_{j}\right)$ of $\mathfrak{g}_{+}$and $\mathfrak{g}_{-}$. The structure coefficients $c_{i j}^{k}$ are those of the Lie algebra $\mathbf{k}$, the coefficients $f_{k}^{i j}$ vanish, and the coefficients $\phi^{i j k}$ are given by the formula $\phi^{i j k}=\frac{1}{4} h^{i l} h^{j m} c_{l m}^{k}$.

Given $x \in \mathfrak{g}$, denote its projection onto $\mathfrak{g}_{ \pm}$by $x_{ \pm}$, and write $\bar{x}=x_{+}-x_{-}$. Writing $x_{+}=p^{i} x_{i}$ and $x_{-}=q_{i} x^{i}$, the condition that $x \in \mathfrak{g}_{0}$ is expressed by the formulas

$$
p^{i} f_{i}^{j k}=q_{i} c_{j k}^{i}=q_{i} \phi^{i j k}=0 .
$$

The element $\rho=\left[x_{i}, x^{i}\right] \in \mathfrak{g}$ is given by the explicit formula

$$
\rho=f_{j}^{j i} x_{i}+c_{i j}^{j} x^{i}
$$

It is easily seen that $\rho \in \mathfrak{g}_{0}$.

Introduce the notations $\operatorname{ad}\left(x_{i}\right) x_{j}=c_{i j}^{k} x_{k}$ and $\operatorname{ad}^{*}\left(x^{i}\right) x_{j}=f_{j}^{k i} x_{k}$ for the adjoint action of $\mathfrak{g}_{+}$on itself, and for the "action" of $\mathfrak{g}_{-}$on $\mathfrak{g}_{+} ; \operatorname{ad}\left(x_{+}\right)$is a derivation of the Lie algebra $\mathfrak{g}_{+}$, while $\operatorname{ad}^{*}\left(x_{-}\right)$is provided $x_{-}$is orthogonal to $\left[\mathfrak{g}_{+}, \mathfrak{g}_{+}\right]$, in particular, if $x_{-} \in \mathfrak{g}_{0}$.

Let $D: \mathfrak{g}_{+} \rightarrow \mathfrak{g}_{+}$denote the derivation $D=-\operatorname{ad}\left(\rho_{+}\right)-\operatorname{ad}^{*}\left(\rho_{-}\right)$of the Lie algebra $\mathfrak{g}_{+}$, given by the explicit formula

$$
D x_{i}=\left(f_{k}^{j k} c_{j i}^{l}+c_{j k}^{k} f_{i}^{j l}\right) x_{l} .
$$

Lemma 2.2. $D x_{i}=f_{i}^{k l} c_{k l}^{j} x_{j}$ and $\operatorname{Tr}(D)=-(\rho, \rho)=-2\left(\rho_{+}, \rho_{-}\right)$

Proof. Let $A: \mathfrak{g}_{+} \otimes \mathfrak{g}_{-} \rightarrow \mathbb{R}$ be the bilinear form $A(x, y)=-\operatorname{Tr}\left(\operatorname{ad}(x) \operatorname{ad}^{*}(y)\right)$, with matrix $A_{i}^{j}=c_{i l}^{k} f_{k}^{j l}$. Summing over the indices $i$ and $l$ in the formula

$$
c_{m k}^{i} f_{l}^{j m}-c_{m l}^{i} f_{k}^{j m}-c_{m k}^{j} f_{l}^{i m}+c_{m l}^{j} f_{k}^{i m}=c_{k l}^{m} f_{m}^{i j}
$$

we see that

$$
-A_{k}^{j}-c_{m i}^{i} f_{k}^{j m}-c_{m k}^{j} f_{i}^{i m}-c_{l m}^{j} f_{k}^{l m}+A_{k}^{j}=0 .
$$

The first and last terms cancel, and we obtain the formula for $D x_{i}$. Taking the trace over the indices $j$ and $k$, we obtain the formula for $\operatorname{Tr}(D)$. 
Denote by $\langle-,-\rangle$ the Killing form of $\mathfrak{g}$, and by $\langle-,-\rangle_{+}$the Killing form of $\mathfrak{g}_{+}$. The proofs of the following formulas are straightforward:

$$
\left\langle x_{i}, x_{j}\right\rangle=2\left\langle x_{i}, x_{j}\right\rangle_{+}, \quad\left\langle x_{i}, x^{j}\right\rangle=-D_{i}^{j}-2 A_{i}^{j}, \quad\left\langle x^{i}, x^{j}\right\rangle=2 f_{l}^{i k} f_{k}^{j l}+c_{k l}^{i} \phi^{j k l}+c_{k l}^{j} \phi^{i k l} .
$$

We see that $\left\langle x_{i}, x^{i}\right\rangle=-3 \operatorname{Tr}(D)$ and $\operatorname{Tr}\left(D^{2}\right)=\frac{1}{2}\langle\rho, \rho\rangle$.

Consider the Manin triple $\left(\mathfrak{g}=\mathbf{k} \oplus \mathbf{l}, \mathfrak{g}_{+}, \mathfrak{g}_{-}\right)$. Identify the weights

$$
\rho(\mathbf{k})=\frac{1}{2} \sum_{\alpha \in \Delta_{+}} \alpha \quad \text { and } \quad \rho(\mathbf{l})=\frac{1}{2} \sum_{\alpha \in \tilde{\Delta}_{+}} \alpha
$$

with elements of $\mathfrak{h}$ using the restriction of the inner product $(-,-)$ to $\mathfrak{h}$. From the explicit basis introduced above for $\mathfrak{g}=\mathbf{k} \oplus \mathbf{l}$, it follows that

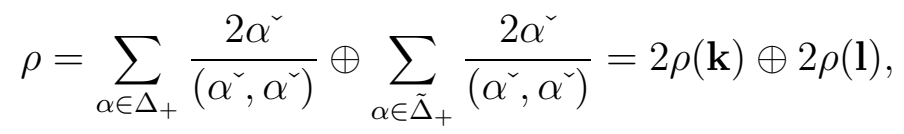

so that $\rho_{ \pm}=(\rho(\mathbf{k}) \pm \rho(\mathbf{l})) \oplus( \pm \rho(\mathbf{k})+\rho(\mathbf{l}))$, and

$$
\operatorname{Tr}(D)=-2(\rho(\mathbf{k}), \rho(\mathbf{k}))+2(\rho(\mathbf{l}), \rho(\mathbf{l})) .
$$

In the case that $\mathbf{p}=\mathbf{b}$, Freudenthal's formula

$$
\frac{(\rho, \rho)}{(\theta, \theta)}=\frac{h \operatorname{dim} \mathbf{k}}{24},
$$

where $\theta$ is the highest root of $\mathbf{k}$, shows that

$$
\operatorname{Tr}(D)=-\frac{h \operatorname{dim} \mathbf{k}}{6(k+h)} .
$$

Finally, we recall Drinfeld's twisting operation [5]. Given a Manin pair $\left(\mathfrak{g}, \mathfrak{g}_{+}, \mathfrak{g}_{-}\right)$and a linear map $R: \mathfrak{g}_{i} \rightarrow \mathfrak{g}_{+}$, we define a subspace

$$
R \cdot \mathfrak{g}_{-}=\left\{x-R x \mid x \in \mathfrak{g}_{-}\right\} .
$$

Introducing the matrix elements $R x^{i}=R^{i j} x_{j}$, the condition that $R \cdot \mathfrak{g}_{-}$is isotropic is equivalent to the matrix $R^{i j}$ being antisymmetric. Taking as our dual bases of $\mathfrak{g}_{+}$and $R \cdot \mathfrak{g}_{-}$the vectors $x_{i}$ and $x^{i}-R^{i j} x_{j}$, we see that the structure coefficients of the Manin pair are modified in the following way:

$$
\begin{aligned}
c_{i j}^{k} & \rightarrow c_{i j}^{k}, \\
f_{k}^{i j} & \rightarrow f_{k}^{i j}-R^{i l} c_{k l}^{j}-R^{l j} c_{k l}^{i}, \\
\phi^{i j k} & \rightarrow \phi^{i j k}+R^{i l} f_{l}^{j k}-R^{j l} f_{l}^{i k}-R^{i l} R^{j m} c_{l m}^{k} .
\end{aligned}
$$

We may illustrate this construction with the Manin triple with $\mathfrak{g}=\mathbf{k} \oplus \mathbf{k}$. Then $\mathfrak{g}_{+}$ has basis

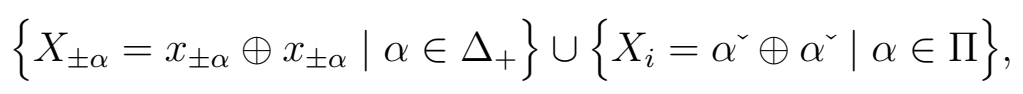


while $\mathfrak{g}_{-}$has the dual basis

$$
\left\{X^{ \pm \alpha}= \pm \frac{2 x_{\mp \alpha}}{\left(\alpha^{\llcorner}, \alpha^{\llcorner}\right)} \mid \alpha \in \Delta_{+}\right\} \cup\left\{X^{i}=\frac{1}{2}\left(\omega_{i} \oplus-\omega_{i}\right) \mid 1 \leq i \leq r\right\} .
$$

The twist

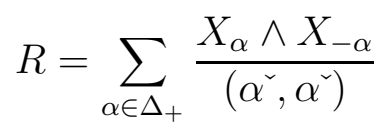

transforms this Manin triple to the Manin pair for which $\mathfrak{g}_{-}=\{(x,-x) \mid x \in \mathbf{k}\}$.

\section{Finite-Dimensional TOPOLOGICAL FIELD THEORY}

In this section, we describe a finite-dimensional analogue of the construction of the next section; this section may be skipped, since its results are not used elsewhere in this paper.

Let $A$ be a graded commutative algebra. A zeroth order differential operator is an operator which commutes with elements of $A$, while an $n$-th order differential operator on $A$ is an operator $L$ whose commutator with elements of $A$ is an $(n-1)$-th order differential operator. A first order derivation is a derivation in the usual sense, while an $n$-th order derivation on $A$ is an operator $L$ such that $[L, a]-L a$ is an $(n-1)$-th order derivation for all $a \in A$ (see Penkava-Schwartz [18]). In particular, $n$-th order derivations are $n$-th order differential operators. A second-order derivation is an operator satisfying the formula

$$
\begin{aligned}
\Delta(a b c) & =\Delta(a b) c+(-1)^{|\Delta||a|} a \Delta(b c)+(-1)^{(|a|+|\Delta|)|b|} b \Delta(a c) \\
& -(\Delta a) b c-(-1)^{|\Delta||a|} a(\Delta b) c-(-1)^{|\Delta|(|a|+|b|)} a b(\Delta c) .
\end{aligned}
$$

Definition 3.1. A Batalin-Vilkovisky algebra is a graded commutative algebra $A$ together with a second-order derivation $\Delta$ of degree -1 such that $\Delta^{2}=0$.

A differential Batalin-Vilkovisky algebra $(A, \Delta, \delta)$ is a Batalin-Vilkovisky algebra together with a derivation $\delta$ of degree 1 on $A$ such that $\delta^{2}=0$, and such that $\delta \Delta+\Delta \delta$ is a first-order differential operator.

Let $A$ be the exterior algebra $\Lambda \mathbf{k}^{*}$, where $\mathbf{k}$ is a vector space. Given a basis $x_{i}$ of $\mathbf{k}$ with dual basis $x^{i}$ of $\mathbf{k}^{*}$, we denote by $a_{i}$ the operator of degree -1 consisting of contraction with $x_{i}$, and by $a^{i}$ the operation of exterior multiplication by $x^{i}$. These operators satisfy the canonical commutation relations

$$
a^{i} a_{j}+a_{j} a^{i}=\delta_{j}^{i}
$$

An operator $L$ on $A$ is an $n$-th differential operator if it can be written as a sum of monomials each of which is at most $n$-th order in the operators $\left\{a_{i}\right\}$.

A second order derivation $\Delta$ of degree -1 on $A$ has the form

$$
\Delta=\frac{1}{2} f_{k}^{i j} a_{i} a_{j} a^{k}+p^{i} a_{i}
$$


for coefficients $f_{k}^{i j}$ and $p^{i}$ such that $f_{k}^{i j}+f_{k}^{j i}=0$. The author learnt the following lemma from G. Zuckerman.

Lemma 3.2. The formula $\Delta^{2}=0$ holds if and only if $f_{k}^{i j}$ are the structure coefficients of a Lie coalgebra, $f_{m}^{i j} f_{l}^{m k}+f_{m}^{j k} f_{l}^{m i}+f_{m}^{k i} f_{l}^{m j}=0$, and $p^{k} f_{k}^{i j}=0$.

Proof. We calculate

$$
\Delta^{2}=\frac{1}{4} f_{k}^{i j} f_{k^{\prime}}^{i^{\prime} j^{\prime}} a_{i} a_{j}\left(\delta_{i^{\prime}}^{k} a_{j^{\prime}}-\delta_{j^{\prime}}^{k} a_{i^{\prime}}+a_{i^{\prime}} a_{j^{\prime}} a^{k}\right) a^{k^{\prime}}+p^{k} f_{k}^{i j} a_{i} a_{j} .
$$

The term proportional to $a^{k} a^{k^{\prime}}$ vanishes, so that

$$
\Delta^{2}=\frac{1}{2} f_{l}^{m i} f_{m}^{j k} a^{l} a_{i} a_{j} a_{k}+p^{k} f_{k}^{i j} a_{i} a_{j} .
$$

The first term is antisymmetric in $a_{i}, a_{j}$ and $a_{k}$, and we see that its vanishing is precisely the Jacobi rule for the Lie cobracket $x_{i} \mapsto \frac{1}{2} f_{k}^{i j} x_{i} \wedge x_{j}$ on $\mathbf{k}$.

A derivation of degree 1 of $A$ has the form

$$
\delta=\frac{1}{2} c_{i j}^{k} a^{i} a^{j} a_{k},
$$

for coefficients $c_{i j}^{k}$ such that $c_{i j}^{k}+c_{j i}^{k}=0$. The proof of the following lemma is dual to the proof of Lemma 3.2.

Lemma 3.3. The formula $\delta^{2}=0$ holds if and only if $c_{i j}^{k}$ are the structure coefficients of a Lie algebra.

We say that a Lie algebra with cobracket $x_{i} \mapsto \frac{1}{2} f_{k}^{i j} x_{i} \wedge x_{j}$ is a Lie bialgebra if the following identity between the structure coefficients $c_{i j}^{k}$ and $f_{k}^{i j}$ is satisfied:

$$
c_{m k}^{i} f_{l}^{j m}-c_{m l}^{i} f_{k}^{j m}-c_{m k}^{j} f_{l}^{i m}+c_{m l}^{j} f_{k}^{i m}=c_{k l}^{m} f_{m}^{i j} .
$$

This is equivalent to supposing that the data $\left(\mathbf{k} \oplus \mathbf{k}^{*}, \mathbf{k}, \mathbf{k}^{*}\right)$ form a Manin triple, where the inner product on $\mathbf{k} \oplus \mathbf{k}^{*}$ is induced by the duality between $\mathbf{k}$ and $\mathbf{k}^{*}$.

A cocharacter of a Lie coalgebra $\mathbf{k}$ is an element $x=p^{i} a_{i} \in \mathbf{k}$ lying in the kernel of the cobracket of $\mathbf{k}$.

Proposition 3.4. There is a bijection between structures of a Lie bialgebra on $\mathbf{k}$ together with a cocharacter $x \in \mathbf{k}$ and differential Batalin-Vilkovisky structures on the algebra $\Lambda \mathbf{k}^{*}$.

Proof. Write $\Delta=\frac{1}{2} f_{k}^{i j} a^{k} a_{i} a_{j}+p^{i} a_{i}$ and $\delta=\frac{1}{2} c_{i j}^{k} a^{i} a^{j} a_{k}$. We have

$$
\Delta \delta+\delta \Delta=\left(-f_{k}^{i m} c_{l m}^{j}+\frac{1}{4} f_{m}^{i j} c_{k l}^{m}\right) a_{i} a_{j} a^{k} a^{l}+\frac{1}{2} f_{k}^{i l} c_{j l}^{k}\left(a^{j} a_{i}-a_{i} a^{j}\right)+p^{i} c_{i j}^{k} a^{j} a_{k} .
$$

This is a first-order differential operator if and only if the first terms cancel, or equivalently if $\mathbf{k}$ is a Lie bialgebra. 
We will denote the operator $\delta \Delta+\delta \Delta$ by $L$. Note that if $\mathbf{k}$ is a Lie bialgebra,

$$
L=D+\operatorname{ad}(x)+\frac{1}{2} \operatorname{Tr}(D)=-\operatorname{ad}\left(\rho_{+}-x\right)-\operatorname{ad}^{*}\left(\rho_{-}\right)+\frac{1}{2} \operatorname{Tr}(D),
$$

where $D$ is the derivation $D_{i}^{j} a^{i} a_{j}$ of $\mathbf{k}$ introduced in the last section and $x=p^{i} x_{i}$.

The following dictionary allows us to interpret the structure of a differential BatalinVilkovisky algebra on $\Lambda \mathbf{k}^{*}$ and the $N=2$ superconformal algebra associated to a Manin triple:

\begin{tabular}{c|c}
$\mathrm{T}$ & $L$ \\
$\mathrm{G}^{+}$ & $\delta$ \\
$\mathrm{G}^{-}$ & $\Delta$ \\
$\mathrm{J}$ & degree
\end{tabular}

Let $(A, \Delta, \delta)$ be a differential Batalin-Vilkovisky algebra such that the operator $L=$ $\delta \Delta+\Delta \delta$ is semi-simple: the vector space $A$ decomposes into a sum of eigenspaces $A_{\lambda}$ on which $L$ equals $\lambda$. The basic complex of $A$ is the subspace $A_{\text {basic }}$ on which

$$
\Delta a=\Delta \delta a=0 .
$$

This is a subcomplex for the differential $\delta$, whose cohomology is called the basic cohomology of $A$. As is explained in the first section of [9], the equivariant cohomology $H_{S^{1}}^{\bullet}(A)$ is the derived functor of the basic cohomology. It is defined by replacing $A$ by the differential Batalin-Vilkovisky algebra $A[\Omega, \omega]$, of graded polynomials in a variable $\Omega$ of degree 2 and a variable $\omega$ of degree 1 , with operators $\Delta_{\text {tot }}$ and $\delta_{\text {tot }}$

$$
\Delta_{\mathrm{tot}}=\frac{\partial}{\partial \omega}+\Delta, \quad \delta_{\mathrm{tot}}=\Omega \frac{\partial}{\partial \omega}+\delta .
$$

Equivalently, the equivariant cohomology is the cohomology of the space of polynomials $A_{0}[\Omega]$ with respect to the differential $\delta-\Omega \Delta$, where $A_{0}$ is the kernel of $L$. This construction is the analogue in finite dimensions of the coupling of an $N=2$ superconformal model with topological gravity.

If $\left(\mathbf{k} \oplus \mathbf{k}^{*}, \mathbf{k}, \mathbf{k}^{*}\right)$ is a Manin pair, so that $\mathbf{k}^{*}$ is no longer assumed to be a Lie subalgebra, we say that $\mathbf{k}$ is a quasi-Lie bialgebra: this notion is due to Drinfeld [5]. The cobracket $x_{i} \mapsto \frac{1}{2} f_{k}^{i j} x_{i} \wedge x_{j}$ no longer satisfies the Jacobi relation, and hence the operator $\Delta^{2}$ does not vanish: rather, we have the formula

$$
\Delta^{2}+\delta \phi+\phi \delta=0
$$

where $\phi=\frac{1}{6} \phi^{i j k} a_{i} a_{j} a_{k}$. We also have the formulas $\Delta \phi+\phi \Delta=\phi^{2}=0$; we call this structure a quasi-Batalin-Vilkovisky algebra. Note that on the cohomology of $A$ with respect to the differential $\delta$, the operator $\Delta$ induces a Batalin-Vilkovisky structure, since $\Delta^{2}$ is chain equivalent to 0 .

It makes no sense to define the basic cohomology of a quasi-Batalin-Vilkovisky algebra. However, if the action of $L$ is semisimple, we may still define the equivariant cohomology, 
as the basic cohomology of $A[\omega, \Omega]$ with operators

$$
\Delta_{\text {tot }}=\frac{\partial}{\partial \omega}+\Delta-\omega \Delta^{2}-\Omega \phi, \quad \delta_{\text {tot }}=\Omega \frac{\partial}{\partial \omega}+\delta .
$$

It may be shown that the equivariant cohomology is the cohomology of the space of polynomials $A_{0}[\Omega]$ with respect to the differential $\delta-\Omega \Delta+\Omega^{2} \phi$, where $A_{0}$ is the kernel of $L$. We will see in Section 5 that this is a model for the coupling of an $N=1 \frac{1}{2}$ superconformal field theory to topological gravity, and that the coupled theory actually has full $N=2$ superconformal symmetry.

\section{A family of $N=2$ SUPERCONFORMAL FIELD THEORIES}

Let $\left(\mathfrak{g}, \mathfrak{g}_{+}, \mathfrak{g}_{-}\right)$be a Manin pair, with dual bases $x_{i}$ of $\mathfrak{g}_{+}$and $x^{i}$ of $\mathfrak{g}_{-}$. We may rewrite the $N=1$ superconformal model associated to $\mathfrak{g}$ in this basis. The Kac-Moody currents $\left\{J_{i}(z), J^{i}(z)\right\}$ have operator products

$$
\begin{aligned}
& J_{i}(z) \cdot J_{j}(w)=-\frac{\frac{1}{2}\left\langle x_{i}, x_{j}\right\rangle}{(z-w)^{2}}+\frac{c_{i j}^{k} J_{k}(w)}{z-w}, \\
& J_{i}(z) \cdot J^{j}(w)=\frac{\delta_{i}^{j}-\frac{1}{2}\left\langle x_{i}, x^{j}\right\rangle}{(z-w)^{2}}+\frac{f_{i}^{j k} J_{k}(w)+c_{k i}^{j} J^{k}(w)}{z-w}, \\
& J^{i}(z) \cdot J^{j}(w)=-\frac{\frac{1}{2}\left\langle x^{i}, x^{j}\right\rangle}{(z-w)^{2}}+\frac{f_{k}^{i j} J^{k}(w)-\phi^{i j k} J_{k}(w)}{z-w},
\end{aligned}
$$

while the free fermionic fields $a_{i}(z)$ and $a^{i}(z)$ have operator products

$$
\begin{aligned}
& a_{i}(z) \cdot a^{j}(w) \sim \frac{\delta_{i}^{j}}{z-w}, \\
& a_{i}(z) \cdot a_{j}(w) \sim a^{i}(z) \cdot a^{j}(w) \sim 0 .
\end{aligned}
$$

The composite currents $I_{i}(z)$ and $I^{i}(z)$ are given by the formulas

$$
\begin{aligned}
I_{i} & =J_{i}-c_{i j}^{k} a^{j} a_{k}-\frac{1}{2} f_{i}^{j k} a_{j} a_{k}, \\
I^{i} & =J^{i}-f_{k}^{i j} a_{j} a^{k}-\frac{1}{2} c_{j k}^{i} a^{j} a^{k}+\frac{1}{2} \phi^{i j k} a_{j} a_{k} .
\end{aligned}
$$

If $\alpha=p^{i} x_{i}+q_{i} x^{i} \in \mathfrak{g}_{0}$, the fields $\left(\alpha_{ \pm}, I\right)$ are given by the formulas

$$
\left(\alpha_{+}, I\right)=p^{i}\left(J_{i}-c_{i j}^{k} a^{j} a_{k}\right), \quad\left(\alpha_{-}, I\right)=q_{i}\left(J^{i}-f_{k}^{i j} a_{j} a^{k}\right) .
$$

For example, these formulas apply if $\alpha=\rho$.

The field $\mathrm{G}$ of the Kac-Todorov model may decomposed into a sum of three fermionic fields $\mathrm{G}^{+}+\mathrm{G}^{-}+\Phi$, with ghost number repsectively $+1,-1$ and -3 , and given by the formulas

$$
\begin{aligned}
\mathrm{G}^{+} & =J_{i} a^{i}-\frac{1}{2} c_{i j}^{k} a^{i} a^{j} a_{k}, \\
\mathrm{G}^{-} & =J^{i} a_{i}-\frac{1}{2} f_{k}^{i j} a_{i} a_{j} a^{k}, \\
\Phi & =\frac{1}{6} \phi^{i j k} a_{i} a_{j} a_{k} .
\end{aligned}
$$




\section{Lemma 4.1.}

$$
\begin{aligned}
\mathrm{G}^{+}(z) \cdot \mathrm{G}^{-}(w) \sim & \frac{\frac{1}{2} \operatorname{dim} \mathfrak{g}-(\rho, \rho)}{(z-w)^{3}}+\frac{a^{i}(w) a_{i}(w)+(\rho, I(w))}{(z-w)^{2}} \\
& +\frac{\frac{1}{2}(J(w), J(w))+\partial a^{i}(w) a_{i}(w)+\frac{1}{2}(\rho, \partial I(w))}{z-w}, \\
\mathrm{G}^{+}(z) \cdot \mathrm{G}^{+}(w) \sim & 0, \\
\mathrm{G}^{-}(z) \cdot \mathrm{G}^{-}(w) \sim & -\frac{\phi^{i j k}\left(J_{i}(w)-c_{i l}^{m} a^{l}(w) a_{m}(w)\right) a_{j}(w) a_{k}(w)}{z-w}-\frac{c_{k l}^{i} \phi^{j k l} \partial a_{i}(w) a_{j}(w)}{z-w}
\end{aligned}
$$

Proof. (1) To calculate $\mathrm{G}^{+}(z) \cdot \mathrm{G}^{-}(w)$, we first observe that

$$
J_{i}(z) a^{i}(z) \cdot J^{j}(w) a_{j}(w) \sim \frac{A_{3}}{(z-w)^{3}}+\frac{A_{2}}{(z-w)^{2}}+\frac{A_{1}+B_{1}}{(z-w)^{2}},
$$

where

$$
\begin{aligned}
A_{3}= & \frac{1}{2}\left(\operatorname{dim} \mathfrak{g}-\left\langle x_{i}, x^{i}\right\rangle\right)=\frac{1}{2} \operatorname{dim} \mathfrak{g}+\frac{3}{2} \operatorname{Tr}(D), \\
A_{2}= & \left(\delta_{i}^{j}-\frac{1}{2}\left\langle x_{i}, x^{j}\right\rangle\right) a^{i}(w) a_{j}(w)+(\rho, J(w)), \\
= & a^{i}(w) a_{i}(w)+\left(-\frac{1}{2} D_{i}^{j}+A_{i}^{j}\right) a^{i}(w) a_{j}(w)+(\rho, I(w)), \\
A_{1}= & \left(\delta_{i}^{j}-\frac{1}{2}\left\langle x_{i}, x^{j}\right\rangle\right) \partial a^{i}(w) a_{j}(w)+J_{i}(w) J^{i}(w), \\
= & \partial a^{i}(w) a_{i}(w)-\frac{1}{2} D_{i}^{j} a^{i}(w) \partial a_{j}(w)+A_{i}^{j} \partial a^{i}(w) a_{j}(w) \\
& +\frac{1}{2}\left(J_{i}(w) J^{i}(w)+J^{i}(w) J_{i}(w)+(\rho, \partial I(w))\right), \\
B_{1}= & f_{i}^{j k} J_{k}(w) a^{i}(w) a_{j}(w)+c_{k i}^{j} J^{k}(w) a^{i}(w) a_{j}(w) .
\end{aligned}
$$

Here, we have made use of the formulas

$$
J_{i}(z) J^{i}(z)=\frac{1}{2}((J(z), J(z))+(\rho, \partial J(z)))
$$

and

$$
(\rho, \partial I(z))=(\rho, \partial J(z))+D_{i}^{j} a^{i}(z) a_{j}(z) .
$$

The term $B_{1}$ is cancelled by the operator products

$$
\begin{gathered}
J_{i}(z) a^{i}(z) \cdot\left(-\frac{1}{2} f_{l}^{j k} a_{j}(w) a_{k}(w) a^{l}(w)\right) \sim-\frac{f_{i}^{j k} J_{k}(w) a^{i}(w) a_{j}(w)}{z-w}, \\
\left(-\frac{1}{2} c_{j k}^{l} a^{j}(z) a^{k}(z) a_{l}(z)\right) \cdot J^{i}(w) a_{i}(w) \sim-\frac{c_{k j}^{i} J^{k}(w) a^{i}(w) a_{j}(w)}{z-w} .
\end{gathered}
$$

The calculation of the operator product $\mathrm{G}^{+}(z) \cdot \mathrm{G}^{-}(w)$ is completed by the formula

$$
\begin{aligned}
& \left(-\frac{1}{2} c_{i j}^{k} a^{i}(z) a^{j}(z) a_{k}(z)\right) \cdot\left(-\frac{1}{2} f_{r}^{p q} a_{p}(w) a_{q}(w) a^{r}(w)\right) \sim \\
& \quad-\frac{\frac{1}{2} \operatorname{Tr}(D)}{(z-w)^{3}}+\frac{\left(\frac{1}{2} D_{i}^{j}-A_{i}^{j}\right) a^{i}(w) a_{j}(w)}{(z-w)^{2}}+\frac{\frac{1}{2} D_{i}^{j} a^{i}(w) \partial a_{j}(w)}{z-w}-\frac{A_{i}^{j} \partial a^{i}(w) a_{j}(w)}{z-w} .
\end{aligned}
$$


(2) To calculate $\mathrm{G}^{+}(z) \cdot \mathrm{G}^{+}(w)$, we first observe that

$$
J_{i}(z) a^{i}(z) \cdot J_{j}(w) a^{j}(w) \sim \frac{A}{z-w}+\frac{B}{z-w},
$$

where $A=c_{i j}^{k} J_{k}(w) a^{i}(w) a^{j}(w)$ and $B=-\frac{1}{2}\left\langle x_{i}, x_{j}\right\rangle \partial a^{i}(w) a^{j}(w)$. The term $A$ is cancelled by the operator products

$$
\begin{aligned}
& J_{i}(z) a^{i}(z) \cdot\left(-\frac{1}{2} c_{j k}^{l} a^{j}(w) a^{k}(w) a_{l}(w)\right) \sim-\frac{1}{2} \frac{c_{j k}^{i} J_{i}(w) a^{j}(w) a^{k}(w)}{z-w}, \\
& \left(-\frac{1}{2} c_{j k}^{l} a^{j}(z) a^{k}(z) a_{l}(z)\right) \cdot J_{i}(w) a^{i}(w) \sim-\frac{1}{2} \frac{c_{j k}^{i} J_{i}(w) a^{j}(w) a^{k}(w)}{z-w} .
\end{aligned}
$$

The term $B$ is cancelled by the operator product

$$
\left(-\frac{1}{2} c_{j k}^{l} a^{j}(z) a^{k}(z) a_{l}(z)\right) \cdot\left(-\frac{1}{2} c_{j k}^{l} a^{j}(w) a^{k}(w) a_{l}(w)\right) \sim \frac{1}{2} \frac{\left\langle x_{i}, x_{j}\right\rangle \partial a^{i}(w) a^{j}(w)}{z-w} .
$$

(3) To calculate $\mathrm{G}^{-}(z) \cdot \mathrm{G}^{-}(w)$, we first observe that

$$
J^{i}(z) a_{i}(z) \cdot J^{j}(w) a_{j}(w) \sim \frac{A}{z-w}+\frac{B}{z-w}+\frac{C}{z-w},
$$

where $A=-\phi^{i j k} J_{i}(w) a_{j}(w) a_{k}(w), B=f_{k}^{i j} J^{k}(w) a_{i}(w) a_{j}(w)$, and $C=-\frac{1}{2}\left\langle x^{i}, x^{j}\right\rangle \partial a_{i}(w) a_{j}(w)$. The term $B$ is cancelled by the operator products

$$
\begin{aligned}
& J^{l}(z) a_{l}(z) \cdot\left(-\frac{1}{2} f_{k}^{i j} a_{i}(w) a_{j}(w) a^{k}(w)\right) \sim-\frac{1}{2} \frac{f_{k}^{i j} J^{k}(w) a_{i}(w) a_{j}(w)}{z-w}, \\
& \left(-\frac{1}{2} f_{k}^{i j} a_{i}(z) a_{j}(z) a^{k}(z)\right) \cdot J^{l}(w) a_{l}(w) \sim-\frac{1}{2} \frac{f_{k}^{i j} J^{k}(w) a_{i}(w) a_{j}(w)}{z-w} .
\end{aligned}
$$

Next observe that

$$
\left(-\frac{1}{2} f_{k}^{i j} a_{i}(z) a_{j}(z) a^{k}(z)\right) \cdot\left(-\frac{1}{2} f_{n}^{l m} a_{l}(w) a_{m}(w) a^{n}(w)\right) \sim \frac{D}{z-w}+\frac{E}{z-w},
$$

where $D=f_{m}^{i j} f_{l}^{m k} a_{i}(w) a_{j}(w) a_{k}(w) a^{l}(w)$ and $E=f_{l}^{i k} f_{k}^{j l} \partial a_{i}(w) a_{j}(w)$. The proof is completed using the formulas

$$
\begin{aligned}
f_{m}^{i j} f_{l}^{m k}+f_{m}^{j k} f_{l}^{m i}+f_{m}^{k i} f_{l}^{m j} & =c_{l m}^{i} \phi^{j k m}+c_{l m}^{j} \phi^{k i m}+c_{l m}^{k} \phi^{i j m}, \\
\left\langle x^{i}, x^{j}\right\rangle & =2 f_{l}^{i k} f_{k}^{j l}+c_{k l}^{i} \phi^{j k l}+c_{k l}^{j} \phi^{i k l} .
\end{aligned}
$$

Motivated by this lemma, we introduce fields

$$
\begin{aligned}
& \mathrm{J}=a^{i} a_{i}+(\rho, I), \\
& \mathrm{T}=\frac{1}{2}\left(J_{i} J^{i}+J^{i} J_{i}+\partial a^{i} a_{i}-a^{i} \partial a_{i}\right), \\
& \mathrm{F}=\frac{1}{2} \phi^{i j k}\left(J_{i}-c_{i l}^{m} a^{l} a_{m}\right) a_{j} a_{k}+\frac{1}{2} c_{k l}^{i} \phi^{j k l} \partial a_{i} a_{j} .
\end{aligned}
$$


Then the above lemma shows that

$$
\begin{aligned}
& \mathrm{G}^{+}(z) \cdot \mathrm{G}^{-}(w) \sim \frac{\frac{1}{2} \operatorname{dim} \mathfrak{g}-(\rho, \rho)}{(z-w)^{3}}+\frac{\mathrm{J}(w)}{(z-w)^{2}}+\frac{\mathrm{T}(w)+\frac{1}{2} \mathrm{~J}(w)}{z-w}, \\
& \mathbf{G}^{-}(z) \cdot \mathbf{G}^{-}(w) \sim-\frac{2 \mathrm{~F}(w)}{z-w} .
\end{aligned}
$$

It is easy to see that

$$
\mathrm{G}^{+}(z) \cdot \Phi(w) \sim \frac{\mathrm{F}(w)}{z-w} .
$$

To complete the verification of the relations of the $N=1 \frac{1}{2}$ superconformal algebra, we must show that the fields $\mathrm{G}^{ \pm}$and $\Phi$ have charges \pm 1 and -3 . The proof uses the following lemma.

Lemma 4.2. If $\alpha \in \mathfrak{g}_{0}$, the superfields $\Omega^{ \pm}(Z)=\left(\alpha_{\mp}, \mathcal{J}(Z)\right)$ satisfy the hypotheses of Proposition 1.4.

Proof. Let us start with the case of $\Omega^{+}(Z)=A^{+}(z)+\theta B^{+}(z)$, where

$$
A^{+}=q_{i} a^{i}, \quad B^{+}=q_{i}\left(J^{i}-f_{k}^{i j} a_{j} a^{k}\right) .
$$

It may be shown by the same methods as in the proof of Proposition 1.2 that it suffices to prove the following operator products:

$$
\begin{aligned}
& \mathrm{G}^{-}(z) \cdot A^{+}(w) \sim \frac{B^{+}(w)}{z-w}, \\
& \mathrm{G}^{+}(z) \cdot B^{+}(w) \sim \frac{A^{+}(w)}{(z-w)^{2}}+\frac{\partial A^{+}(w)}{z-w}, \\
& \mathrm{G}^{+}(z) \cdot A^{+}(w) \sim \Phi(z) \cdot A^{+}(w) \sim 0 .
\end{aligned}
$$

The vanishing of $q_{i} c_{j k}^{i}$ shows that $\mathrm{G}^{+}(z) \cdot A^{+}(w) \sim 0$, while the vanishing of $q_{i} \phi^{i j k}$ shows that $\Phi(z) \cdot A^{+}(w) \sim 0$. The formula

$$
\mathrm{G}^{-}(z) \cdot a^{i}(w) \sim \frac{J^{i}(w)-f_{k}^{i j} a_{j}(w) a^{k}(w)}{z-w}
$$

shows that $\mathrm{G}^{-}(z) \cdot A^{+}(w) \sim B^{+}(w) /(z-w)$.

To calculate $B^{+}(z) \cdot \mathrm{G}^{+}(w)$, observe that

$$
q_{i} J^{i}(z) \cdot J_{j}(w) a^{j}(w) \sim \frac{A_{2}}{(z-w)^{2}}+\frac{A_{1}+B_{1}}{z-w},
$$

where $A_{2}=\left(\delta_{j}^{i}-\frac{1}{2}\left\langle x^{i}, x_{j}\right\rangle\right) q_{i} a^{j}(w), D=c_{j k}^{i} q_{i} a^{j}(w) J^{k}(w)$ and $E=-f_{j}^{i k} q_{i} a^{j}(w) J_{k}(w)$. The term $B_{1}$ is cancelled by the operator product

$$
q_{i}\left(-f_{j}^{i k} a_{k}(z) a^{j}(z)\right) \cdot J_{l}(w) a^{l}(w) \sim \frac{f_{j}^{i k} q_{i} a^{j}(w) J_{k}(w)}{z-w} .
$$


The calculation of $\mathrm{G}^{+}(z) \cdot B^{+}(w)$ is completed by the operator product

$$
q_{i}\left(-f_{k}^{i j} a_{j}(z) a^{k}(z)\right) \cdot\left(-\frac{1}{2} c_{l m}^{n} a^{l}(w) a^{m}(w) a_{n}(w)\right) \sim \frac{\frac{1}{2}\left\langle x_{j}, x^{i}\right\rangle q_{i} a^{j}(w)}{(z-w)^{2}} .
$$

The case of $\Omega^{-}(Z)=A^{-}(z)+\theta B^{-}(z)$, where

$$
A^{-}=p^{i} a_{i}, \quad B^{-}=p^{i}\left(J_{i}-c_{i j}^{k} a^{j} a_{k}\right),
$$

is proved in a similar way, by proving the formulas

$$
\begin{aligned}
& \mathrm{G}^{+}(z) \cdot A^{-}(w) \sim \frac{B^{-}(w)}{z-w} \\
& \mathrm{G}^{-}(z) \cdot B^{-}(w) \sim \frac{A^{-}(w)}{(z-w)^{2}}+\frac{\partial A^{-}(w)}{z-w} \\
& \mathrm{G}^{-}(z) \cdot A^{-}(w) \sim \Phi(z) \cdot A^{-}(w) \sim 0 .
\end{aligned}
$$

Using this lemma, we see that

$$
\begin{aligned}
\mathrm{G}^{+}(z) \cdot(\rho, I(w)) & \sim \mathrm{G}^{+}(z) \cdot\left(\rho_{+}, I(w)\right)+\mathrm{G}^{+}(z) \cdot\left(\rho_{-}, I(w)\right) \\
& \sim \frac{\left(\rho_{+}, a(w)\right)}{(z-w)^{2}}+\frac{\left(\rho_{+}, \partial a(w)\right)}{z-w} \\
\mathrm{G}^{-}(z) \cdot(\rho, I(w)) & \sim \mathrm{G}^{-}(z) \cdot\left(\rho_{+}, I(w)\right)+\mathrm{G}^{-}(z) \cdot\left(\rho_{-}, I(w)\right) \\
& \sim \frac{\left(\rho_{-}, a(w)\right)}{(z-w)^{2}}+\frac{\left(\rho_{-}, \partial a(w)\right)}{z-w} .
\end{aligned}
$$

Furthermore,

$$
\begin{aligned}
& a^{i}(z) a_{i}(z) \cdot \mathrm{G}^{+}(w) \sim \frac{c_{i j}^{j} a^{i}(w)}{(z-w)^{2}}+\frac{c_{i j}^{j} \partial a^{i}(w)}{z-w} \sim \frac{\left(\rho_{+}, a(w)\right)}{(z-w)^{2}}+\frac{\left(\rho_{+}, \partial a(w)\right)}{z-w}, \\
& a^{i}(z) a_{i}(z) \cdot \mathrm{G}^{-}(w) \sim \frac{f_{j}^{i j} a_{i}(w)}{(z-w)^{2}}+\frac{f_{j}^{i j} \partial a_{i}(w)}{z-w} \sim \frac{\left(\rho_{-}, a(w)\right)}{(z-w)^{2}}+\frac{\left(\rho_{-}, \partial a(w)\right)}{z-w} .
\end{aligned}
$$

Combining these formulas, we see that $\mathrm{J}(z) \cdot \mathrm{G}^{ \pm}(w) \sim \pm \mathrm{G}^{ \pm}(w) /(z-w)$.

To complete the verification of the $N=1 \frac{1}{2}$ superconformal algebra, we must check that $\mathrm{G}^{-}(z) \cdot \Phi(w) \sim 0$, which is a consequence of the formula

$$
f_{m}^{i l} \phi^{j k m}+f_{m}^{j l} \phi^{k l m}+f_{m}^{k l} \phi^{i j m}=0,
$$

and that

$$
J(z) \cdot \Phi(w) \sim-\frac{3 \Phi(w)}{z-w}
$$

which follows from the fact that $(\rho, I(z)) \cdot \Phi(w) \sim 0$.

Having constructed an action of the $N=1 \frac{1}{2}$ superconformal algebra, it is simple to construct others: we simply apply Proposition 1.4 with

$$
\Omega^{ \pm}(z)=\left(\alpha_{\mp}, \mathcal{J}(z)\right),
$$


where $\alpha=\alpha_{+}+\alpha_{-}$is an element of $\mathfrak{g}_{0}$. This has the effect of modifying the fields to

$$
\begin{aligned}
\mathrm{T} & =\frac{1}{2}\left(J_{i} J^{i}+J^{i} J_{i}+\partial a^{i} a_{i}-a^{i} \partial a_{i}+(\alpha, \partial I)\right), \\
\mathrm{G}^{+} & =J_{i} a^{i}-\frac{1}{2} c_{i j}^{k} a^{i} a^{j} a_{k}+q_{i} \partial a^{i}, \\
\mathrm{G}^{-} & =J^{i} a_{i}-\frac{1}{2} f_{k}^{i j} a_{i} a_{j} a^{k}+p^{i} \partial a_{i}, \\
\mathrm{~J} & =a^{i} a_{i}+(\rho+\bar{\alpha}, I),
\end{aligned}
$$

while $\Phi$ and $\mathrm{F}$ are unchanged. The central charge becomes $d=\frac{1}{2} \operatorname{dim} \mathfrak{g}-(\rho, \rho)-(\alpha, \alpha)$.

There is an especially natural choice for $\alpha$, namely $\alpha=-\bar{\rho}$. With this value of $\alpha$, the field $\mathrm{J}$ takes the especially simple form $a^{i} a_{i}$ of the ghost number, while the central charge becomes simply $d=\frac{1}{2} \operatorname{dim} \mathfrak{g}$. It is natural to restrict $\alpha$ to lie in the lattice for which the zero-mode of the field $\mathrm{J}$ has integral eigenvalues: this is equivalent to the zero-mode of the field $(\rho+\bar{\alpha}, I(z))$ having integral eigenvalues.

\section{Coupling to topological gravity}

There is a modification of the $N=1 \frac{1}{2}$ superconformal algebra with twisted boundary conditions, parametrized by an angle $\theta$, in which the fields $\mathrm{G}^{ \pm}(z)$ and $\Phi(z)$ satisfy the boundary conditions

$$
\begin{aligned}
\mathrm{G}^{ \pm}\left(e^{2 \pi i} z\right) & =e^{ \pm i \theta} \mathrm{G}^{ \pm}(z), \\
\Phi\left(e^{2 \pi i} z\right) & =e^{-3 i \theta} \Phi(z) .
\end{aligned}
$$

We may realize the $N=1 \frac{1}{2}$ superconformal algebra with these boundary conditions by using the construction of the last section but with fermions having boundary conditions

$$
a^{k}\left(e^{2 \pi i} z\right)=e^{i \theta} a^{k}(z) \quad, \quad a_{k}\left(e^{2 \pi i} z\right)=e^{-i \theta} a_{k}(z) .
$$

Of these boundary conditions, only the cases where $e^{i \theta}= \pm 1$ have an underlying $N=1$ superconformal symmetry generated by the field $\mathrm{G}(z)=\mathrm{G}^{+}(z)+\mathrm{G}^{-}(z)+\Phi(z)$ : the choice of periodic boundary conditions $e^{i \theta}=1$ goes by the name of the Ramond sector, while the choice of anti-periodic boundary conditions $e^{i \theta}=-1$ is called the Neveu-Schwarz sector.

The twisting construction of Witten and Eguchi-Yang is a way of constructing a topological field theory in its Ramond (Neveu-Schwarz) sector from an $N=1 \frac{1}{2}$ theory in its Neveu-Schwarz (Ramond) sector. The Hilbert spaces of the two theories, are identical, and all that is changed is the stress-energy tensor $\mathrm{T}(z)$, which is replaced by its twist $\mathrm{T}_{\text {top }}(z)=\mathrm{T}(z)+\frac{1}{2} \partial \mathrm{J}(z)$. This has the effect of modifying the conformal dimensions of the fields in the theory, subtracting from them half of their $\mathrm{U}(1)$ charge. In particular, the field $\mathrm{G}^{+}(z)$ now has conformal dimension 1 . In the Ramond sector of the topological theory, this allows us to think of the zero mode $\mathrm{Q}$ of the field $\mathrm{G}^{+}$as a differential on the space of states of the theory, and the zero mode of the field $\mathrm{J}$ as a degree operator. (The operator $\mathrm{Q}$ may be identified with the mode $\mathrm{G}_{1 / 2}^{+}$of the untwisted $N=2$ theory.) 
The operator $Q$ raises degree by 1 , and its cohomology is by definition the space of physical states of the topological field theory. By the formula

$$
\mathrm{T}_{\text {top }}(z)=\left[\mathrm{Q}, \mathrm{G}^{-}(z)\right]
$$

we see that the Virasoro algebra acts trivially on this cohomology space, explaining why this model is called a topological field theory. In the model of the last section, this differential

$$
\mathrm{Q}=\operatorname{Res}\left(J_{i} a^{i}-\frac{1}{2} c_{i j}^{k} a^{i} a^{j} a_{k}\right)
$$

is the BRS operator which calculates the semi-infinite cohomology for the projective action of the infinite dimensional Lie algebra $\widehat{\mathfrak{g}_{+}}$acting through the currents $J_{i}(z)$. Note that this differential is independent of $\alpha \in \mathfrak{g}_{0}$, and hence so is the physical Hilbert space. By contrast, the zero mode of $J$, which defines the grading, and the zero-mode of the spin 2 field $\mathrm{G}^{-}$do depend on $\alpha$.

In [9], we have described the coupling of a topological field theory associated to an $N=2$ superconformal field theory to topological gravity. This may be generalized to the case of an $N=1 \frac{1}{2}$ superconformal action. The semi-infinite Weil complex $W$ is the Fock space associated to a pair $\{\gamma(z), \beta(z)\}$ of free bosonic fields and a pair $\{c(z), b(z)\}$ of free fermionic fields, with operator products

$$
b(w) \cdot c(z) \sim \beta(z) \cdot \gamma(w) \sim \frac{1}{z-w},
$$

while $c(z) \cdot c(w) \sim b(z) \cdot b(w) \sim \gamma(z) \cdot \gamma(w) \sim \beta(z) \cdot \beta(w) \sim 0$. The vacuum $|-1\rangle$ of $W$ is characterized by its being annihilated by the modes

$$
\left\{b_{n}, \beta_{n} \mid n \geq-1\right\} \cup\left\{c_{n}, \gamma_{n} \mid n>1\right\} ;
$$

this Fock space is called the (-1)-picture.

The couple theory is the tensor product of the highest weight module $\mathcal{E}$ of the $N=1 \frac{1}{2}$ superconformal algebra with the semi-infinite Weil complex $W$, and has an action of the $N=2$ superconformal algebra, with central charge $d-3$, given by the formulas

$$
\begin{aligned}
\mathbb{T}_{\text {top }} & =2 \partial c b+c \partial b+2 \partial \gamma \beta+\gamma \partial \beta+\mathrm{T}_{\text {top }}, \\
\mathbb{G}^{+} & =\gamma b+\mathrm{G}^{+}, \\
\mathbb{G}^{-} & =b+2 \partial c \beta+c \partial \beta+\mathrm{G}^{-}+c \mathbf{F}-\gamma \Phi, \\
\mathbb{J} & =c b+2 \gamma \beta+\mathrm{J} .
\end{aligned}
$$

It is interesting that on coupling to topological gravity, we recover a full $N=2$ superconformal symmetry, despite only having $N=1 \frac{1}{2}$ superconformal symmetry in the matter sector.

Suppose that $\mathcal{E}$ is the vector space of states of an $N=1 \frac{1}{2}$ superconformal field theory: thus, it has two commuting actions of the $N=1 \frac{1}{2}$ superconformal algebra, corresponding to chiral and anti-chiral sectors of the conformal field theory. The cohomology $H^{\bullet}(\mathcal{E})$ 
of the differential $\mathrm{Q}+\overline{\mathrm{Q}}$ is a commutative algebra, with respect to the fusion product, while the operator $\mathrm{G}_{0}^{-}-\overline{\mathrm{G}}_{0}^{-}$induces an operator $\Delta$ on $H^{\bullet}(\mathcal{E})$ giving it the structure of a Batalin-Vilkovisky algebra.

Define the equivariant cohomology $H_{S^{1}}(\mathcal{E})$ of $\mathcal{E}$ to be the semi-relative cohomology of the coupled theory $W \otimes \bar{W} \otimes \mathcal{E}$, that is, the cohomology of the BRS operator $\mathbb{Q}+\overline{\mathbb{Q}}$ on the kernel of the difference of zero-modes $\mathbb{G}_{0}^{-}-\overline{\mathbb{G}}_{0}^{-}$. This is the space of physical states of the topological field theory underlying $\mathcal{E}$ to topological gravity. The equivariant cohomology $H_{S^{1}}^{\bullet}(\mathcal{E})$ is a gravity algebra, just as in the case of an $N=2$ superconformal field theory.

The equivariant cohomology of the model introduced in the last section is independent of $\alpha_{-} \in \mathfrak{g}_{0}$, and we conjecture that it is independent of $\alpha_{+}$as well, in the sense that there is a flat (or, possibly, projectively flat) connection on the bundle over $\mathfrak{g}_{+} \cap \mathfrak{g}_{0}$ whose fibre at $\alpha_{+}$is the equivariant cohomology of the corresponding model.

Define the field

$$
\mathbb{K}=c\left(\partial c \beta+\mathrm{G}^{-}-\gamma \Phi\right)
$$

of conformal dimension 1 and charge 0 ; thus, the fields $\mathbb{T}_{\text {top }}$ and $\mathbb{J}$ are invariant under the operation $e^{\operatorname{ad}(\mathbb{K})}$ of conjugation by the exponential of the zero mode of $\mathbb{K}$.

\section{Proposition 5.1.}

$$
\begin{aligned}
e^{\operatorname{ad}(\mathbb{K})} \mathbb{G}^{+}= & c \partial c b+c\left(2 \partial \gamma \beta+\gamma \partial \beta+\mathrm{T}_{\text {top }}\right) \\
& +\mathrm{G}^{+}+\gamma\left(b-\mathrm{G}^{-}\right)+\gamma^{2} \Phi-\partial(c \mathbb{J})+\frac{d-3}{2} \partial^{2} c, \\
e^{\operatorname{ad}(\mathbb{K})} \mathbb{G}^{-}= & b .
\end{aligned}
$$

Proof. The formula for $e^{\operatorname{ad}(\mathbb{K})} \mathbb{G}^{+}$follows from the formulas

$$
\begin{aligned}
\operatorname{ad}(\mathbb{K}) \mathbb{G}^{+}= & c \partial c b+c\left(2 \partial \gamma \beta+\gamma \partial \beta+\mathrm{T}_{\text {top }}\right) \\
& +\mathrm{G}^{+}-\gamma \mathbf{G}^{-}+\gamma^{2} \Phi-\partial(c \mathbb{J})+\frac{d-3}{2} \partial^{2} c-c \gamma \mathbf{F}, \\
\frac{1}{2} \operatorname{ad}(\mathbb{K})^{2} \mathbb{G}^{+}= & c \gamma \mathbf{F}-c \partial c \gamma \Phi, \\
\frac{1}{6} \operatorname{ad}(\mathbb{K})^{3} \mathbb{G}^{+}= & c \partial c \gamma \Phi, \quad \operatorname{ad}(\mathbb{K})^{4} \mathbb{G}^{+}=0 .
\end{aligned}
$$

The formula for $e^{\operatorname{ad}(\mathbb{K})} \mathbb{G}^{-}$follows from the formulas

$$
\begin{aligned}
\operatorname{ad}(\mathbb{K}) \mathbb{G}^{+} & =-2 \partial c \beta-c \partial \beta-\mathrm{G}^{-}-2 c \mathrm{~F}+\gamma \Phi+c \partial c \Phi, \\
\frac{1}{2} \operatorname{ad}(\mathbb{K})^{2} \mathbb{G}^{+} & =c \mathrm{~F}-2 c \partial c \Phi \\
\frac{1}{6} \operatorname{ad}(\mathbb{K})^{3} \mathbb{G}^{+} & =c \partial c \Phi, \quad \operatorname{ad}(\mathbb{K})^{4} \mathbb{G}^{+}=0 . \quad \square
\end{aligned}
$$

We may now prove the following extension of Theorem 2.2 of [9].

Theorem 5.2. The equivariant cohomology $H_{S^{1}}^{\bullet}(\mathcal{E})$ is naturally isomorphic to the cohomology of the complex $\mathcal{E}_{0}[\Omega]$ of polynomials in a variable $\Omega$ of ghost number 2 with 
coefficients in the kernel $\mathcal{E}_{0}$ of the rotation operator $\left(\mathrm{T}_{\text {top }}\right)_{0}-\left(\overline{\mathrm{T}}_{\text {top }}\right)_{0}$, with respect to the differential

$$
(\mathrm{Q}+\overline{\mathrm{Q}})-\Omega\left(\mathrm{G}_{0}^{-}-\overline{\mathrm{G}}_{0}^{-}\right)+\Omega^{2}\left(\Phi_{0}+\bar{\Phi}_{0}\right)
$$

Proof. The operator

$$
k c_{-k} \beta_{k}+\mathrm{G}_{0}^{-}
$$

has degree -1 , and commutes with the operators $\left(\mathbb{T}_{\text {top }}\right)_{0}$ and $\mathbb{G}_{0}^{-}$; in proving this, we use the formula $\left[\mathrm{G}_{n}^{-}, \mathrm{F}_{m}\right]=(2 n-m) \Phi_{n+m}$. Thus, it restricts to a map of the basic complex $(W \otimes \bar{W} \otimes \mathcal{E})_{\text {basic }}$ to itself. Thus, we may calculate the equivariant cohomology by restricting attention to the kernel of the commutator

$$
\left[\mathbb{G}_{0}^{+}+\overline{\mathbb{G}}_{0}^{+}, k c_{-k} \beta_{k}+\mathrm{G}_{0}^{-}\right]=k\left(c_{-k} b_{k}+\gamma_{-k} \beta_{k}\right)+\left(\mathrm{T}_{\text {top }}\right)_{0},
$$

acting on the basic subcomplex. Taking $k=0$, we see that we may suppose that $\left(\mathrm{T}_{\text {top }}\right)_{0}=$ 0 . For $k<-1$, the operators $c_{-k} b_{k}$ and $\gamma_{-k} \beta_{k}$ are negative, and we may suppose they each vanish. For $k>0$ or $k=-1$, the operators $c_{-k} b_{k}$ and $\gamma_{-k} \beta_{k}$ are positive, and we may again suppose they vanish. Similar arguments apply in the anti-chiral sector, so we may calculate the equivariant cohomology on the subcomplex spanned by the vectors $\left\{c_{0}^{m} \bar{c}_{0}^{\bar{m}} \gamma_{0}^{n} \bar{\gamma}_{0}^{\bar{n}}|-1\rangle \mid m, \bar{m} \in\{0,1\}, n, \bar{n} \geq 0\right\} \otimes\left\{v \in \mathcal{E} \mid\left(\mathrm{T}_{\text {top }}\right)_{0} v=\left(\overline{\mathrm{T}}_{\text {top }}\right)_{0} v=\left(\mathrm{G}_{0}^{-}-\overline{\mathrm{G}}_{0}^{-}\right) v=0\right\}$.

The rest of the proof is as in [9].

\section{TWISTING}

Given an element $R \in \Lambda^{2} \mathfrak{g}_{+}$, we have shown in Section 2 how to twist a Manin pair $\left(\mathfrak{g}, \mathfrak{g}_{+}, \mathfrak{g}_{-}\right)$, to obtain a new Manin pair in which the space $\mathfrak{g}_{-}$is replaced by $R \cdot \mathfrak{g}_{-}$, spanned by the vectors $x^{i}-R^{i j} x_{j}$. In this section, we describe how the $N=1 \frac{1}{2}$ superconformal symmetry is modified by twisting.

If $R^{i j}$ is the antisymmetric matrix defining a twist, introduce the operator $\mathrm{R}=$ $\frac{1}{2} R^{i j} a_{i} a_{j}$. The following formulas are easily proved:

$$
\begin{aligned}
& \mathrm{G}^{+}(z) \cdot \mathrm{R}(w) \sim \frac{\frac{1}{2} c_{i j}^{k} R^{i j} a_{k}(w)-q_{i} R^{i j} a_{k}(w)}{(z-w)^{2}}-\frac{R^{i j} a_{i}(w)\left(J_{j}(w)-c_{j k}^{l} a^{k}(w) a_{l}(w)\right)}{z-w} \\
&+\frac{\frac{1}{2} c_{i j}^{k} R^{i j} \partial a_{k}(w)}{(z-w)^{2}}, \\
& \mathrm{G}^{-}(z) \cdot \mathrm{R}(w) \sim-\frac{\frac{1}{2} f_{l}^{i j} R^{k l} a_{i}(w) a_{j}(w) a_{k}(w)}{z-w} .
\end{aligned}
$$

We modify the fermionic fields in the following way: $\tau_{\mathrm{R}} \mathrm{G}^{+}=\mathrm{G}^{+}$, while

$$
\begin{aligned}
\tau_{\mathrm{R}} \mathrm{G}^{-} & =\mathrm{G}^{-}+\left[\mathrm{G}^{+} \mathrm{R}\right]_{1}, \\
\tau_{\mathrm{R}} \Phi & =\Phi-\frac{2}{3}\left[\mathrm{G}^{-} \mathrm{R}\right]_{1}+\frac{1}{6}\left[\left[\mathrm{G}^{+} \mathrm{R}\right]_{1} \mathrm{R}\right]_{1} .
\end{aligned}
$$


Proposition 6.1. The twisted fields $\tau_{\mathrm{R}} \mathrm{G}^{ \pm}$and $\tau_{\mathrm{R}} \Phi$ are the fields associated to the twisted Manin pair $\left(\mathfrak{g}, \mathfrak{g}_{+}, R \cdot \mathfrak{g}_{-}\right)$, with $\alpha_{+}$replaced by

$$
\tau_{\mathrm{R}} \alpha_{+}=\alpha_{+}-\frac{1}{2} c_{i j}^{k} R^{i j} x_{k}
$$

while $\alpha_{-}$is unchanged. They generate an action of the $N=1 \frac{1}{2}$ superconformal algebra if and only if

$$
\operatorname{ad}\left(\tau_{\mathrm{R}} \alpha_{+}\right) R^{l m}+\frac{1}{2} f_{k}^{l m} c_{i j}^{k} R^{i j}=\operatorname{ad}^{*}\left(\alpha_{-}\right) R=0 .
$$

The twists of the other fields are given by the formulas

$$
\begin{aligned}
\tau_{\mathrm{R}} \mathrm{J} & =\mathrm{J}-\left[\mathrm{G}^{+}\left[\mathrm{G}^{+} \mathrm{R}\right]_{2}\right]_{1}, \\
\tau_{\mathrm{R}} \mathrm{T} & =\mathrm{T}-\frac{1}{2} \partial\left[\mathrm{G}^{+}\left[\mathrm{G}^{+} \mathrm{R}\right]_{2}\right]_{1}, \\
\tau_{\mathrm{R}} \mathrm{F} & =\mathrm{F}-\frac{2}{3}\left[\mathrm{G}^{+}\left[\mathrm{G}^{-} \mathrm{R}\right]_{1}\right]_{1}+\frac{1}{6}\left[\mathrm{G}^{+}\left[\left[\mathrm{G}^{+} \mathrm{R}\right]_{1} \mathrm{R}\right]_{1}\right]_{1} .
\end{aligned}
$$

Proof. Since twisting sends the coefficients $f_{k}^{i j}$ to

$$
f_{k}^{i j}-R^{i l} c_{k l}^{j}-R^{l j} c_{k l}^{i}
$$

and the vector $x^{i}$ to $x^{i}-R^{i j} x_{j}$, it twists the field $J^{i} a_{i}-\frac{1}{2} f_{k}^{i j} a_{i} a_{j} a^{k}$ to

$$
\left(J^{i}-R^{i j} J_{k}\right) a_{i}-\frac{1}{2} f_{k}^{i j} a_{i} a_{j} a^{k}+R^{i l} c_{k l}^{j} a_{i} a_{j} a_{k}=J^{i} a_{i}-\frac{1}{2} f_{k}^{i j} a_{i} a_{j} a^{k}-R^{i j} a_{i}\left(J_{j}-c_{j k}^{l} a^{k} a_{l}\right),
$$

while leaving the field $q_{i} \partial a^{i}$ invariant. This shows that $\mathrm{G}^{-}+\left[\mathrm{G}^{+} \mathrm{R}\right]_{1}$ equals the field $\mathrm{G}^{-}$ associated to the twisted Manin triple $\left(\mathfrak{g}, \mathfrak{g}_{+}, R \cdot \mathfrak{g}_{-}\right)$, but with $\alpha_{+}$replaced by $\tau_{\mathrm{R}} \alpha_{+}$.

Twisting sends the coefficients $\phi^{i j k}$ to

$$
\phi^{i j k}+R^{i l} f_{l}^{j k}-R^{j l} f_{l}^{i k}-R^{i l} R^{j m} c_{l m}^{k}
$$

The formulas

$$
\begin{aligned}
\left(R^{i l} f_{l}^{j k}-R^{j l} f_{l}^{i k}\right) a_{i} a_{j} a_{k} & =-4\left[\mathrm{G}^{-} \mathrm{R}\right]_{1}, \\
R^{i l} R^{j m} c_{l m}^{k} a_{i} a_{j} a_{k} & =\left[\left[\mathrm{G}^{+} \mathrm{R}\right]_{1} \mathrm{R}\right]_{1}
\end{aligned}
$$

show that the field $\tau_{\mathrm{R}} \Phi$ associated to the twisted Manin triple $\left(\mathfrak{g}, \mathfrak{g}_{+}, R \cdot \mathfrak{g}_{-}\right)$equals $\Phi-$ $\frac{2}{3}\left[\mathrm{G}^{-} \mathrm{R}\right]_{1}+\frac{1}{6}\left[\left[\mathrm{G}^{+} \mathrm{R}\right]_{1} \mathrm{R}\right]_{1}$.

The twisted field $\tau_{\mathrm{R}} \mathrm{J}$ is given by the formula

$$
\tau_{\mathrm{R}} \mathrm{J}=\left[\mathrm{G}^{+} \tau_{\mathrm{R}} \mathrm{G}^{-}\right]_{2}=\mathrm{J}+\left[\mathrm{G}^{+}\left[\mathrm{G}^{+} \mathrm{R}\right]_{1}\right]_{2}=\mathrm{J}-\left[\mathrm{G}^{+}\left[\mathrm{G}^{+} \mathrm{R}\right]_{2}\right]_{1} .
$$

The twisted stress-energy tensor $\tau_{\mathrm{R}} \mathrm{T}$ is given by the formula

$$
\tau_{\mathrm{R}} \mathrm{T}=\left[\mathrm{G}^{+} \tau_{\mathrm{R}} \mathrm{G}^{-}\right]_{1}-\frac{1}{2} \partial \tau_{\mathrm{R}} \mathrm{J}=\mathrm{T}+\left[\mathrm{G}^{+}\left[\mathrm{G}^{+} \mathrm{G}^{-}\right]_{1}\right]_{1}+\frac{1}{2} \partial\left[\mathrm{G}^{+}\left[\mathrm{G}^{+} \mathrm{R}\right]_{2}\right]_{1} .
$$

However, $\left[\mathrm{G}^{+}\left[\mathrm{G}^{+} \mathrm{G}^{-}\right]_{1}\right]_{1}=0$, while $\partial\left[\mathrm{G}^{+}\left[\mathrm{G}^{+} \mathrm{R}\right]_{2}\right]_{1}=\left[\mathrm{G}^{+} \partial\left[\mathrm{G}^{+} \mathrm{R}\right]_{2}\right]_{1}$. The formula for $\tau_{\mathrm{R}} \mathrm{F}$ follows from the fact that $\tau_{\mathrm{R}} \mathrm{F}=\left[\mathrm{G}^{+} \tau_{\mathrm{R}} \Phi\right]_{1}$.

The twisted fields $\tau_{\mathrm{R}} \mathrm{G}^{ \pm}$and $\tau_{\mathrm{R}} \Phi$ generate an action of the $N=1 \frac{1}{2}$ superconformal algebra provided the following three conditions hold:

(1) $\tau_{\mathrm{R}} \alpha_{+} \perp\left[R \cdot \mathfrak{g}_{-}, R \cdot \mathfrak{g}_{-}\right]$; 
(2) $\alpha_{-} \perp\left[\mathfrak{g}_{+}, \mathfrak{g}_{+}\right]$;

(3) $\alpha_{-} \perp\left[R \cdot \mathfrak{g}_{-}, R \cdot \mathfrak{g}_{-}\right]$.

The first condition may be rewritten

$$
\left(p^{k}-\frac{1}{2} c_{l m}^{k} R^{l m}\right)\left(f_{k}^{i j}-R^{i l} c_{k l}^{j}+R^{j l} c_{k l}^{i}\right)=0 .
$$

Since $p^{k} f_{k}^{i j}$ vanishes and

$$
\left(p^{k}-\frac{1}{2} c_{l m}^{k} R^{l m}\right)\left(R^{i l} c_{k l}^{j}+R^{l j} c_{k l}^{i}\right)=\operatorname{ad}\left(\alpha_{+}\right) R,
$$

the condition on $\alpha_{+}$follows.

The second of these conditions is automatically satisfied, while the third may be rewritten

$$
q_{k}\left(R^{i l} f_{l}^{j k}-R^{j l} f_{l}^{i k}-R^{i l} R^{j m} c_{l m}^{k}\right)=q_{k}\left(R^{i l} f_{l}^{j k}-R^{j l} f_{l}^{i k}\right)=0,
$$

where we have used the fact that, since $\alpha_{-} \in \mathfrak{g}_{0}, q_{k} c_{i j}^{k}=0$. But this equation is equivalent to $\operatorname{ad}^{*}\left(\alpha_{-}\right) R=0$.

From this proposition, we see that the twist does not change the BRS operator $\mathrm{Q}$, and only changes the field $\mathrm{J}$ by a BRS commutator $\left[\mathrm{Q},\left[\mathrm{G}^{+} \mathrm{R}\right]_{2}\right]$. Thus, the cohomology of the space of physical states, and its grading, is unchanged. Since the field $\mathrm{G}^{-}$is changed by a BRS commutator $[\mathrm{Q}, \mathrm{R}]$, the action of the Batalin-Vilkovisky operator $\Delta$ on the cohomology is unchanged.

The equivariant cohomology is also left unchanged by a twist, since the field $\mathbb{G}^{+}=$ $\gamma \beta+\mathrm{G}^{+}$is unchanged, while $\mathbb{G}^{-}$changes by a BRS commutator:

$$
\tau_{\mathrm{R}} \mathbb{G}^{-}=\mathbb{G}^{-}+\left[\mathbb{Q}, \mathrm{R}+\frac{2}{3} c\left[\mathrm{G}^{-} \mathrm{R}\right]_{1}-\frac{1}{6} c\left[\left[\mathrm{G}^{+} \mathrm{R}\right]_{1} \mathrm{R}\right]_{1}\right]
$$

\section{The SL(2)/SL(2) MODEL}

In this section, we study two $N=2$ superconformal actions, associated to the two conjugacy classes of parabolic subalgebras of the Lie algebra $\mathbf{k}=\mathbf{s l}(2), \mathbf{p}=\mathbf{b}$ and $\mathbf{p}=\mathbf{k}$. We will construct an intertwining operator between these two superconformal algebras, or rather between certain $N=1 \frac{1}{2}$ twists of them, in the sense of the last section. Aharony et al. [2] have conjectured that similar results hold for any simple Lie algebra k: for example, that for any simple Lie algebra $\mathbf{k}$, there is an explicit intertwining operator between the $N=1 \frac{1}{2}$ superconformal action associated to the Manin pair based on $\mathbf{k} \oplus \mathbf{k}$ and the corresponding action for the Manin triple based on $\mathbf{k} \oplus \mathfrak{h}$, with $\alpha=-\bar{\rho}$.

Let us start with a very simple example of a Manin triple: the two-dimensional abelian Lie algebra with hyperbolic inner product $\mathbb{R}^{1,1}$. Let $\left\{x_{+}\right\}$be a basis of $\mathfrak{g}_{+} \cong \mathbb{R}$, and let $x^{+}$be the vector such that $\left(x_{+}, x^{+}\right)=1$. We introduce a pair of free fermionic fields $\left\{a_{+}(z), a^{+}(z)\right\}$, such that

$$
a_{+}(z) \cdot a^{+}(w) \sim \frac{1}{z-w}
$$


and a pair of free bosonic fields $\{\gamma, \beta\}$ such that

$$
\beta(z) \cdot \gamma(w) \sim \frac{1}{z-w}
$$

We may realize the Heisenberg algebra $\widehat{\mathbb{R}^{1,1}}$ in a Fock space associated to the fields $\gamma(z)$ and $\beta(z)$ by means of currents $J_{+}=\beta$ and $J^{+}=\partial \gamma$. In this way, we obtain a free-field realization of the $N=2$ superconformal algebra with central charge $d=1$ :

$$
\begin{aligned}
\mathrm{G}^{+} & =a^{+} \beta, \\
\mathrm{G}^{-} & =a_{+} \partial \gamma, \\
\mathrm{J} & =a^{+} a_{+}, \\
\mathrm{T} & =\partial \gamma \beta+\frac{1}{2}\left(\partial a^{+} a_{+}-a^{+} \partial a_{+}\right) .
\end{aligned}
$$

The associated BRS operator $\mathrm{Q}=\operatorname{Res}\left(a_{+} \beta\right)$ has one-dimensional cohomology, spanned by the vacuum |\rangle . (Physicists call the Fock space associated to the fields $\left\{\gamma, \beta, a^{+}, a_{+}\right\}$ a Kugo-Ojima quartet, while mathematicians call it a Koszul complex.)

Next, we turn to the the Manin triple $\mathfrak{g}=\mathbf{s l}(2) \oplus \mathbf{s o}(2)$ associated to the Borel subalgebra $\mathbf{b}$ of $\mathbf{s l}(2)$. The Lie algebra $\mathbf{s l}(2)$ has basis

$$
J_{+}=\left(\begin{array}{ll}
0 & 1 \\
0 & 0
\end{array}\right), \quad J_{0}=\left(\begin{array}{cc}
1 & 0 \\
0 & -1
\end{array}\right), \quad J_{-}=\left(\begin{array}{ll}
0 & 0 \\
1 & 0
\end{array}\right)
$$

Denote by $\kappa$ the inner product $\left(J_{+}, J_{-}\right)$: since the dual Coxeter number of $\mathbf{s l}(2)$ is 2 , this Manin triple corresponds to a representation of $\widehat{\operatorname{sl}(2)}$ at level $k=\kappa-2$. Denote by $j$ the element of $\mathbf{s o}(2)$ corresponding to the element $J_{0} \in \mathbf{s l}(2)$. The subalgebra $\mathfrak{g}_{+}$has basis

$$
x_{0}=\frac{1}{2}\left(J_{0}+j\right), \quad x_{-}=J_{-},
$$

while the dual basis of the subalgebra $\mathfrak{g}_{-}$is

$$
x^{0}=(2 \kappa)^{-1}\left(J_{0}-j\right), \quad x^{-}=\kappa^{-1} J_{+} .
$$

The element $\rho$ equals $\left[x_{0}, x^{0}\right]+\left[x_{-}, x^{-}\right]=-\kappa^{-1} x_{0}-x^{0}$, so that $\rho_{+}=-\kappa^{-1} x_{0}$ and $\rho_{-}=-x^{0}$.

Let $J_{ \pm}(z)$ and $J_{0}(z)$ be fields realizing a highest weight representation of $\widehat{\operatorname{sl}(2)}$ at level $k=\kappa-2$ :

$$
\begin{aligned}
& J_{+}(z) \cdot J_{-}(w) \sim \frac{k}{(z-w)^{2}}+\frac{J_{0}(w)}{z-w} \\
& J_{0}(z) \cdot J_{ \pm}(w) \sim \pm \frac{2 J_{ \pm}(w)}{z-w} \\
& J_{0}(z) \cdot J_{0}(w) \sim \frac{2 k}{(z-w)^{2}} .
\end{aligned}
$$


The field $j(z)$ is a free field with

$$
j(z) \cdot j(w) \sim-\frac{2 \kappa}{(z-w)^{2}} .
$$

Introduce free fermionic fields $\left\{a_{0}(z), a^{0}(z), a_{-}(z), a^{-}(z)\right\}$, with

$$
a_{0}(z) \cdot a^{0}(w) \sim a_{-}(z) \cdot a^{-}(w) \sim \frac{1}{z-w} .
$$

Consider the following two-parameter family of actions of the $N=2$ superconformal algebra, with central charge $d=3-(1-s t) / \kappa$ :

$$
\begin{aligned}
\mathrm{G}^{+}= & a^{+} \beta+a^{0}\left(\frac{1}{2}\left(J_{0}+j\right)+a^{-} a_{-}\right)+a^{-} J_{-}+s \partial a^{0}, \\
\mathrm{G}^{-}= & a_{+} \partial \gamma+\kappa^{-1} a_{0}\left(\frac{1}{2}\left(J_{0}-j\right)+a^{-} a_{-}\right)+\kappa^{-1} a_{-} J_{+}+t \partial a_{0}, \\
\mathrm{~J}= & \left.a^{+} a_{+}+a^{0} a_{0}+a^{-} a_{-}-\kappa^{-1}(1+s)\left[\mathrm{Q}, a_{0}\right]-(1-t)\left[\mathrm{Q}, a^{0}\right]\right), \\
\mathrm{T}= & \beta \partial \gamma+(2 \kappa)^{-1}\left(\frac{1}{2}\left(J_{0}\right)^{2}+J_{+} J_{-}+J_{-} J_{+}-\frac{1}{2} j^{2}+s\left[\mathrm{Q}, \partial a_{0}\right]+\kappa t\left[\mathrm{Q}, \partial a^{0}\right]\right), \\
& +\frac{1}{2}\left(\partial a^{0} a_{0}+\partial a^{0} a_{0}+\partial a^{-} a_{-}-a^{0} \partial a_{0}-a^{0} \partial a_{0}-a^{-} \partial a_{-}\right) .
\end{aligned}
$$

Here we have parametrized the element $\alpha \in \mathfrak{g}_{0}$ as $\alpha=s x_{0}+\kappa t x^{0}$, the BRS operator $\mathrm{Q}=\operatorname{Res}\left(\mathrm{G}^{+}\right)$is the zero-mode of the field $\mathrm{G}^{+}$, and

$$
\begin{aligned}
{\left[\mathrm{Q}, a_{0}\right] } & =\frac{1}{2}\left(J_{0}+j\right)+a^{-} a_{-}, \\
\kappa\left[\mathrm{Q}, a^{0}\right] & =\frac{1}{2}\left(J_{0}-j\right)+a^{-} a_{-} .
\end{aligned}
$$

For $s=t=0$, this is the tensor product of the actions of the $N=2$ superconformal algebra associated to the abelian Lie algebra $\mathbb{R}^{1,1}$ and the Kazama-Suzuki model at level $k$, associated to the Manin triple $\mathbf{s l}(2) \oplus \mathbf{s o}(2)$. We will show that at $s=1$ and $t=-1$, this representation of the $N=2$ superconformal algebra is equivalent to that associated to the Manin triple $\mathbf{s l}(2) \oplus \mathbf{s l}(2)$ : according to Karabali and Schnitzer [13] and Schnitzer [19], this describes the $\mathrm{SL}(2) / \mathrm{SL}(2)$ model at level $k$.

Consider the effect of twisting this action by the operator $\mathrm{R}=(2 \kappa)^{-1} a_{+} a_{-}$. The conditions of Proposition 6.1 are satisfied, and we obtain an action of the $N=1 \frac{1}{2}$ superconformal algebra where the fields $\mathrm{G}^{+}, \mathrm{J}$ and $\mathrm{T}$ are unchanged by the twist, while

$$
\begin{aligned}
\tau_{\mathrm{R}} \mathrm{G}^{-}= & (2 \kappa)^{-1}\left(a_{+}\left(J_{-}+2 \kappa \partial \gamma\right)+a_{0}\left(J_{0}-j+2 a^{-} a_{-}\right)+a_{-}\left(2 J_{+}-\beta\right)\right. \\
& \left.+a^{0} a_{+} a_{-}+2 \partial a_{0}\right), \\
\Phi=- & \frac{1}{3 \kappa^{2}} a_{+} a_{0} a_{-} .
\end{aligned}
$$

Of course, $F=[Q, \Phi]$ : we will not need its explicit formula.

We now introduce the field

$$
\mathbf{A}=\gamma\left(J_{+}-2 a^{-} a_{0}+a^{0} a_{+}\right) .
$$


The following operator products are easily demonstrated:

$$
\begin{aligned}
\mathrm{T}(z) \cdot \mathrm{A}(w) & \sim \frac{\mathrm{A}(w)}{(z-w)^{2}}+\frac{\partial \mathrm{A}(w)}{z-w}-(s+t) \frac{\partial\left(\gamma(w)\left(J_{+}(w)-2 a^{-}(w) a_{0}(w)\right)\right)}{(z-w)^{2}} \\
\mathrm{~J}(z) \cdot \mathrm{A}(w) & \sim(t-s-2) \frac{\gamma(w)\left(J_{+}(w)-2 a^{-}(w) a_{0}(w)\right)}{z-w} .
\end{aligned}
$$

In order for conjugation by the zero-mode of the field $A$ to be physically meaningful, the field A should have conformal dimension 1 and charge 0 . For this reason, we set $s=-1$ and $t=1$ for the remainder of this section. For these values of the parameters, the fields $\mathrm{J}$ and $\mathrm{T}$ are given by the formulas

$$
\begin{aligned}
\mathrm{J}= & a^{+} a_{+}+a^{0} a_{0}+a^{-} a_{-}, \\
\mathrm{T}= & \beta \partial \gamma+(4 \kappa)^{-1}\left(\left(J_{0}\right)^{2}+2 J_{+} J_{-}+2 J_{-} J_{+}-j^{2}-2 \partial j\right), \\
& +\frac{1}{2}\left(\partial a^{0} a_{0}+\partial a^{0} a_{0}+\partial a^{-} a_{-}-a^{0} \partial a_{0}-a^{0} \partial a_{0}-a^{-} \partial a_{-}\right)
\end{aligned}
$$

The Wakimoto representation of the affine Kac-Moody algebra at level $-k-4$ is realized in the Fock space of the free bosonic field $j(z)$ and free bosonic fields $\beta(z)$ and $\gamma(z)$, with currents

$$
\begin{aligned}
& K_{+}=\beta \\
& K_{0}=-2 \gamma \beta+j \\
& K_{-}=-\gamma^{2} \beta+\gamma j-(k+4) \partial \gamma .
\end{aligned}
$$

(The highest weight $\Lambda$ of the Wakimoto representation equals the vacuum expectation value of the zero-mode of $j$.)

\section{Theorem 7.1.}

$$
\begin{aligned}
& e^{-\operatorname{ad}(\mathrm{A})} \mathrm{G}^{+}=a^{+}\left(J_{+}+K_{+}\right)+a^{0}\left(\frac{1}{2}\left(J_{0}+K_{0}\right)-a^{+} a_{+}+a^{-} a_{-}\right)+a^{-}\left(J_{-}+K_{-}\right) \\
&-2 a^{+} a^{-} a_{0}, \\
& e^{-\operatorname{ad}(\mathrm{A})} \tau_{\mathrm{R}} \mathrm{G}^{-}=(2 \kappa)^{-1}\left(a_{+}\left(J_{-}-K_{-}\right)+a_{0}\left(J_{0}-K_{0}\right)+a_{-}\left(J_{+}-K_{+}\right)\right),
\end{aligned}
$$

while the fields J, T and $\Phi$ are left invariant by the automorphism $e^{-\operatorname{ad}(\mathrm{A})}$.

Proof. Denote the field $J_{+}-2 a^{-} a_{0}+a^{0} a_{+}$by $I_{+}$. The calculation of $e^{-\operatorname{ad}(\mathrm{A})} \mathrm{G}^{+}$follows from the formulas

$$
\begin{aligned}
\operatorname{ad}(\mathrm{A}) \mathrm{G}^{+} & =-a^{+} I_{+}-a^{0} \gamma\left(I_{+}-\beta\right)+a^{-}(-\gamma j+(k+4) \partial \gamma)+2 \partial a^{-} \gamma-\partial a^{0}, \\
\frac{1}{2} \operatorname{ad}(\mathrm{A})^{2} \mathrm{G}^{+} & =-a^{0} \gamma I_{+}+a^{-} \gamma^{2}\left(I_{+}-\beta\right)+2 \partial a^{-} \gamma, \\
\frac{1}{6} \operatorname{ad}(\mathrm{A})^{3} \mathrm{G}^{+} & =a^{-} \gamma^{2} I_{+}, \quad \operatorname{ad}(\mathrm{A})^{4} \mathrm{G}^{+}=0 .
\end{aligned}
$$


The calculation of $e^{-\operatorname{ad}(\mathrm{A})} \tau_{\mathrm{R}} \mathrm{G}^{-}$follows from the formulas

$$
\begin{aligned}
\operatorname{ad}(\mathrm{A}) \tau_{\mathrm{R}} \mathrm{G}^{-} & =(2 \kappa)^{-1}\left(a_{+}(\gamma j+k \partial \gamma)+2 a_{0} \gamma\left(I_{+}-\beta\right)+a_{-} I_{+}-2 \partial a_{+} \gamma+2 \partial a_{0}\right), \\
\frac{1}{2} \operatorname{ad}(\mathrm{A})^{2} \tau_{\mathrm{R}} \mathrm{G}^{-} & =(2 \kappa)^{-1}\left(a_{+} \gamma^{2}\left(\beta-I_{+}\right)+2 a_{0} \gamma I_{+}-2 \partial a_{+} \gamma\right), \\
\frac{1}{6} \operatorname{ad}(\mathrm{A})^{3} \tau_{\mathrm{R}} \mathrm{G}^{-} & =-(2 \kappa)^{-1} a_{+} \gamma^{2} I_{+}, \quad \operatorname{ad}(\mathrm{A})^{4} \tau_{\mathrm{R}} \mathrm{G}^{-}=0 .
\end{aligned}
$$

The fields $\mathrm{J}$ and $\mathrm{T}$ are invariant under $\operatorname{ad}(\mathrm{A})$, since we have chosen $s=-1$ and $t=1$, while the invariance of the field $\Phi$ is easily seen to satisfy $\operatorname{ad}(\mathrm{A}) \Phi=0$.

As a last transformation, let us apply the twist $\tau_{-R}$ to the the above action of the $N=1 \frac{1}{2}$ superconformal algebra. Once more, the conditions of Proposition 6.1 are satisfied. The resulting action actually has $N=2$ superconformal symmetry, with action

$$
\begin{aligned}
& \mathbb{G}^{+}= e^{-\operatorname{ad}(\mathrm{A})} \mathrm{G}^{+}=a^{+}\left(J_{+}+K_{+}\right)+a^{0}\left(\frac{1}{2}\left(J_{0}+K_{0}\right)-a^{+} a_{+}+a^{-} a_{-}\right)+a^{-}\left(J_{-}+K_{-}\right) \\
&-2 a^{+} a^{-} a_{0}, \\
& \mathbb{G}^{-}=\left.\tau_{-\mathrm{R}} e^{\mathrm{ad}(-\mathrm{A})} \tau_{\mathrm{R}} \mathrm{G}^{-}=\kappa^{-1}\left(-a_{+} K_{-}+a_{0}\left(\frac{1}{2}\left(J_{0}-K_{0}\right)+a^{+} a_{+}+a^{-} a_{-}\right)+a_{-} J_{+}+\partial a_{0}\right)\right), \\
& \mathbb{T}=\mathrm{T}+(2 \kappa)^{-1}\left[\mathbb{Q}, \partial a_{0}\right], \\
& \mathbb{J}=\mathrm{J}+\kappa^{-1}\left[\mathbb{Q}, a_{0}\right],
\end{aligned}
$$

where $\mathbb{Q}$ is the zero mode of $\mathbb{G}^{+}$, and

$$
\left[\mathbb{Q}, a_{0}\right]=\frac{1}{2}\left(J_{0}-K_{0}\right)-a^{+} a_{+}+a^{-} a_{-} .
$$

This is the $N=2$ action associated to the Manin triple $\mathfrak{g}=\mathbf{s l}(2) \oplus \mathbf{s l}(2)$ : $\mathfrak{g}_{+}$has basis

$$
x_{+}=J_{+}+K_{+}, \quad x_{0}=\frac{1}{2}\left(J_{0}+K_{0}\right), \quad x_{1}=J_{-}+K_{-},
$$

$\mathfrak{g}_{-}$has dual basis

$$
x^{+}=-\kappa^{-1} K_{-}, \quad x^{0}=(2 \kappa)^{-1}\left(J_{0}-K_{0}\right), \quad x^{-}=\kappa^{-1} J_{+},
$$

the subspace $\mathfrak{g}_{0}$ is spanned by $x_{0}$, and the element $\alpha$ is taken to equal $\kappa^{-1} x_{0}$.

\section{Appendix. The operator product expansion and vertex algebras}

In this paper, we have made free use of the operator product expansion of conformal field theory, as axiomatized in the notion of a vertex algebra. A vertex algebra is a $\mathbb{Z} / 2$ graded vector space $V$, together with an even map $\partial: V \rightarrow V$ (which may be interpreted as differentiation), and a sequence of bilinear products

$$
[A B]_{n}: V \otimes V \rightarrow V, \quad n \in \mathbb{Z},
$$


such that for given $A$ and $B$, the product $[A B]_{n}$ vanishes for $n$ sufficiently large. These products are the Laurent coefficients of the operator product of the two fields in twodimensional conformal field theory (Belavin-Polyakov-Zamolodchikov [3]):

$$
A(z) \cdot B(w)=\sum_{n=-\infty}^{\infty} \frac{[A B]_{n}(w)}{(z-w)^{n}} .
$$

For this reason, elements of a vertex algebra are sometimes referred to as fields. The product $[A B]_{0}$ is called the normal product of $A$ and $B$, and should be thought of as a renormalized product on $V$.

These products satisfy axioms, first written down by Borcherds [4], which are reminiscent of those of those of a Lie algebra. (We have reindexed his products in order to agree with the conventions of physicists: thus, we write $[A B]_{n}$ where Borcherds writes $A_{n-1} B$.)

\section{(Jacobi)}

$$
\left[[A B]_{m} C\right]_{n}=\sum_{i=0}^{\infty}(-1)^{i}\left(\begin{array}{c}
m-1 \\
i
\end{array}\right)\left(\left[A[B C]_{n+i}\right]_{m-i}+(-1)^{m+|A||B|}\left[B[A C]_{i+1}\right]_{m+n-i-1}\right)
$$

Here $\left(\begin{array}{l}a \\ i\end{array}\right)=a(a-1) \ldots(a-i+1) / i !$

(Commutativity)

$$
[B A]_{n}=(-1)^{n+|A||B|} \sum_{i=0}^{\infty} \frac{(-1)^{i}}{i !} \partial^{i}[A B]_{n+i}
$$

(Identity) There is an even element 1 such that $\partial 1=0$ and for all $A \in V$,

$$
[1 A]_{n}= \begin{cases}A, & n=0, \\ 0, & n \neq 0 .\end{cases}
$$

For $m=n=0$, the Jacobi rule simply says that

$$
\left[[A B]_{0} C\right]_{n}=\sum_{i \leq 0}\left[A[B C]_{-i}\right]_{i}+\sum_{i>0}(-1)^{|A||B|}\left[B[A C]_{i}\right]_{-i} .
$$

Subtracting the corresponding formula for $(-1)^{|A||B|}\left[[B A]_{0} C\right]_{0}$, we see that

$$
\left[[A B]_{0} C\right]_{n}-(-1)^{|A||B|}\left[[B A]_{0} C\right]_{0}=\left[A[B C]_{0}\right]_{0}-(-1)^{|A||B|}\left[B[A C]_{0}\right]_{0} .
$$

The above axioms imply the following properties of the derivation $\partial$.

Proposition A.1. $[(\partial A) B]_{n}=(1-n)[A B]_{n-1}$ and $\partial[A B]_{n}=[(\partial A) B]_{n}+[A(\partial B)]_{n}$ Proof. To calculate $[(\partial A) B]_{n}$, we use the Jacobi rule, and the formula $\partial A=[A 1]_{-1}$ :

$$
[(\partial A) B]_{n}=\left[[A 1]_{-1} B\right]_{n}=\sum_{i=0}^{\infty}(-1)^{i}\left(\begin{array}{c}
-2 \\
i
\end{array}\right)\left(\left[A[1 B]_{n+i}\right]_{-i-1}-\left[1[A B]_{i+1}\right]_{n-i-2}\right) .
$$


Note that $(-1)^{i}\left(\begin{array}{c}-2 \\ i\end{array}\right)=i+1$. If $n<1$, only the first term on the left-hand side contributes, with $i=-n$. On the other hand, if $n>1$, only the second term contributes, with $i=n-2$.

To calculate $[A(\partial B)]_{n}$, we apply the commutativity axiom:

$$
\begin{aligned}
{[A(\partial B)]_{n} } & =\sum_{i=0}^{\infty} \frac{(-1)^{n+i+|A||B|}}{i !} \partial^{i}[(\partial B) A]_{n+i} \\
& =\sum_{i=0}^{\infty} \frac{(-1)^{n+i+|A||B|}}{i !}(1-n-i) \partial^{i}[B A]_{n+i-1} \\
& =(n-1) \sum_{i=0}^{\infty} \frac{(-1)^{(n-1)+i+|A||B|}}{i !} \partial^{i}[B A]_{n+i-1}+\sum_{i=0}^{\infty} \frac{(-1)^{n+i+|A||B|}}{i !} \partial^{i}[B A]_{n+i} \\
& =(n-1)[A B]_{n-1}+\partial[A B]_{n} . \quad
\end{aligned}
$$

In this paper, we will only use the following consequence of the Jacobi rule, discovered by Sevrin et al. [21]. This formula shows the extent to which the map $B \mapsto[A B]_{m}$, $m>0$, behaves like a derivation.

Proposition A.2. If $m>0$,

$$
\begin{aligned}
{\left[A[B C]_{n}\right]_{m} } & =\sum_{i=0}^{m-1}\left(\begin{array}{c}
m-1 \\
i
\end{array}\right)\left[[A B]_{m-i} C\right]_{n+i}+(-1)^{|A||B|}\left[B[A C]_{m}\right]_{n} \\
& =\sum_{i=0}^{\infty} \frac{(-1)^{i}}{i !}\left[\left[\left(\partial^{i} A\right) B\right]_{m} C\right]_{n+i}+(-1)^{|A||B|}\left[B[A C]_{m}\right]_{n} .
\end{aligned}
$$

Proof. If $m>0$, the Jacobi rule may be rewritten

$$
\left[[A B]_{m} C\right]_{n}=\sum_{i=0}^{m-1}(-1)^{i}\left(\begin{array}{c}
m-1 \\
i
\end{array}\right)\left(\left[A[B C]_{n+i}\right]_{m-i}-(-1)^{|A||B|}\left[B[A C]_{m-i}\right]_{n+i}\right) .
$$

It follows that

$$
\begin{aligned}
\sum_{j=0}^{m-1}\left(\begin{array}{c}
m-1 \\
j
\end{array}\right)\left[[A B]_{m-j} C\right]_{n+j}= & \sum_{j=0}^{m-1} \sum_{i=0}^{m-j-1}(-1)^{i}\left(\begin{array}{c}
m-1 \\
j
\end{array}\right)\left(\begin{array}{c}
m-j-1 \\
i
\end{array}\right) \\
& \left(\left[A[B C]_{n+i+j}\right]_{m-i-j}-(-1)^{|A||B|}\left[B[A C]_{m-i-j}\right]_{n+i+j}\right) .
\end{aligned}
$$

The proposition now follows from the combinatorial identity

$$
\sum_{\{0 \leq i, j \leq m-1 \mid i+j=k\}}(-1)^{i}\left(\begin{array}{c}
m-1 \\
j
\end{array}\right)\left(\begin{array}{c}
m-j-1 \\
i
\end{array}\right)= \begin{cases}1, & k=0, \\
0, & k>0,\end{cases}
$$

which is a trivial consequence of the identity $(x+(1-x))^{m-1}=1$.

The singular part of the operator product expansion is denoted

$$
A(z) \cdot B(w) \sim \sum_{n>0} \frac{[A B]_{n}(w)}{(z-w)^{n}} .
$$


We will call the algebraic structure similar to a vertex algebra, but with products $[A B]_{n}$ only for $n>0$, satisfying the commutativity and identity axioms as well as the formula of Propositions A.2 and A.1, a chiral algebra. The reader is warned that this is not a standard piece of terminology, but it will be useful to have a word for this notion.

A stress-energy tensor in a vertex algebra is an even element $\mathrm{T}$ such that for all $A \in V$,

$$
[\mathrm{T} A]_{1}=\partial A
$$

and for some scalar $c$ (the central charge of $\mathrm{T}$ ),

$$
\mathrm{T}(z) \cdot \mathrm{T}(w) \sim \frac{\frac{1}{2} c \cdot 1(w)}{(z-w)^{4}}+\frac{2 \mathrm{~T}(w)}{(z-w)^{2}}+\frac{\partial \mathrm{T}(w)}{z-w} .
$$

It is usual to denote the scalar multiples $c \cdot 1(w)$ of the identity 1 simply by $c$. An element $A$ of $V$ has conformal dimension $a \in \mathbb{R}$ if $[\mathrm{T} A]_{2}=a A$. For example, the conformal dimension of 1 is zero, while the conformal dimension of $\mathrm{T}$ is 2 .

Lemma A.3. The conformal dimension of $[A B]_{0}$ is the sum of the conformal dimensions of $A$ and $B$.

Proof. By the Leibniz rule, we see that

$$
\left[\mathrm{T}[A B]_{0}\right]_{2}=\left[[\mathrm{T} A]_{2} B\right]_{0}+\left[[\mathrm{T} A]_{1} B\right]_{1}+\left[A[\mathrm{~T} B]_{2}\right]_{0}=(a+b)[A B]_{0}+[(\partial A) B]_{1} .
$$

The second term vanishes by Proposition A.1.

To a chiral algebra $V$ (and in particular to a vertex algebra) is associated a Lie algebra

$$
L(V)=V\left[z, z^{-1}\right] /\left\{(\partial A) z^{n}+n A z^{n-1}\right\},
$$

with bracket

$$
\left[A z^{n}, B z^{m}\right]=\sum_{k \geq 0}\left(\begin{array}{l}
n \\
k
\end{array}\right)[A B]_{k+1} z^{n+m-k} .
$$

If $A$ has conformal dimension $a$, one denotes the element $A z^{n}$ of the Lie algebra $L(V)$ by $A_{n-a+1}$. It follows from the properties of the identity that $1_{n}=0$ for $n \neq 0$, and that $1_{0}$ lies in the centre of $L(V)$. The modes of the stress-energy tensor satisfy the relations of the Virasoro algebra,

$$
\left[\mathrm{T}_{n}, \mathrm{~T}_{m}\right]=(n-m) \mathrm{T}_{n+m}+\frac{c}{12} n\left(n^{2}-1\right) 1_{n+m}
$$

By a representation of a chiral algebra, we will mean a representation of its associated Lie algebra $L(V)$. We only consider representations in the category $\mathcal{O}$, the definition of which is analogous to that of the category $\mathcal{O}$ for an affine Kac-Moody algebra or the Virasoro algebra.

If $K$ is a field of conformal dimension 1, then given fields $A$ and $B=[K A]_{1}$, we have the relation $B_{n}=\left[K_{0}, A_{n}\right]$. For this reason, we denote the graded commutator with the zero mode $K_{0}$ by $\operatorname{ad}(K)$. 
An element $A$ is a primary field of conformal dimension $a$ if

$$
\mathrm{T}(z) \cdot A(w) \sim \frac{a A(w)}{(z-w)^{2}}+\frac{\partial A(w)}{z-w} .
$$

The modes of a primary field $A$ in $L(V)$ satisfy the following commutation relations with the Virasoro algebra spanned by $\mathrm{T}_{n}$ :

$$
\left[\mathrm{T}_{n}, A_{m}\right]=((a-1) n-m) A_{n+m} .
$$

We can now give the proof of Proposition 1.2.

Proof. The proof is a straighforward, though lengthy, exercise in the application of Proposition A.2. We will use the formulas

$$
\begin{aligned}
\mathrm{T} & =\left[\mathrm{G}^{+} \mathrm{G}^{-}\right]_{1}-\frac{1}{2} \partial \mathrm{J}=\left[\mathrm{G}^{-} \mathrm{G}^{+}\right]_{1}+\frac{1}{2} \partial \mathrm{J}=\frac{1}{2}\left[\mathrm{G}^{+} \mathrm{G}^{-}\right]_{1}+\frac{1}{2}\left[\mathrm{G}^{-} \mathrm{G}^{+}\right]_{1}, \\
\mathrm{~J} & =\left[\mathrm{G}^{+} \mathrm{G}^{-}\right]_{2}=-\left[\mathrm{G}^{-} \mathrm{G}^{+}\right]_{2}, \\
d & =\left[\mathrm{G}^{+} \mathrm{G}^{-}\right]_{3}=\left[\mathrm{G}^{-} \mathrm{G}^{+}\right]_{3} .
\end{aligned}
$$

(1) We start by calculating $\mathrm{J}(z) \cdot \mathrm{J}(w)$ :

$$
\begin{aligned}
& {[\mathrm{JJ}]_{n}=\left[\mathrm{J}\left[\mathrm{G}^{+} \mathrm{G}^{-}\right]_{2}\right]_{n}=\left[\left[\mathrm{JG}^{+}\right]_{1} \mathrm{G}^{-}\right]_{n+1}+\left[\mathrm{G}^{+}\left[\mathrm{JG}^{-}\right]_{n}\right]_{2}} \\
& = \begin{cases}{\left[\left[\mathrm{JG}^{+}\right]_{1} \mathrm{G}^{-}\right]_{2}+\left[\mathrm{G}^{+}\left[\mathrm{JG}^{-}\right]_{1}\right]_{2}=0,} & n=1, \\
{\left[\left[\mathrm{JG}^{+}\right]_{1} \mathrm{G}^{-}\right]_{3}=d,} & n=2, \\
{\left[\left[\mathrm{JG}^{+}\right]_{1} \mathrm{G}^{-}\right]_{n+1}=0,} & n>2 .\end{cases}
\end{aligned}
$$

(2) Next we calculate $\mathbf{J}(z) \cdot \mathbf{T}(w)$ :

$$
\begin{aligned}
{[\mathrm{JT}]_{n}=} & \frac{1}{2}\left(\left[\mathrm{~J}\left[\mathrm{G}^{+} \mathrm{G}^{-}\right]_{1}\right]_{n}+\left[\mathrm{J}\left[\mathrm{G}^{-} \mathrm{G}^{+}\right]_{1}\right]_{n}\right) \\
= & \frac{1}{2}\left(\left[\left[\mathrm{JG}^{+}\right]_{1} \mathrm{G}^{-}\right]_{n}+\left[\left[\mathrm{JG}^{-}\right]_{1} \mathrm{G}^{+}\right]_{n}\right)+\frac{1}{2}\left(\left[\mathrm{G}^{+}\left[\mathrm{JG}^{-}\right]_{n}\right]_{1}+\left[\mathrm{G}^{-}\left[\mathrm{JG}^{+}\right]_{n}\right]_{1}\right) \\
& = \begin{cases}\frac{1}{2}\left(\left[\left[\mathrm{JG}^{+}\right]_{1} \mathrm{G}^{-}\right]_{1}+\left[\left[\mathrm{JG}^{-}\right]_{1} \mathrm{G}^{+}\right]_{1}+\left[\mathrm{G}^{+}\left[\mathrm{JG}^{-}\right]_{1}\right]_{1}+\left[\mathrm{G}^{-}\left[\mathrm{JG}^{+}\right]_{1}\right]_{1}\right)=0, & n=1, \\
\frac{1}{2}\left(\left[\left[\mathrm{JG}^{+}\right]_{1} \mathrm{G}^{-}\right]_{2}+\left[\left[\mathrm{JG}^{-}\right]_{1} \mathrm{G}^{+}\right]_{2}\right)=\mathrm{J}, & n=2, \\
\frac{1}{2}\left(\left[\left[\mathrm{JG}^{+}\right]_{1} \mathrm{G}^{-}\right]_{3}+\left[\left[\mathrm{JG}^{-}\right]_{1} \mathrm{G}^{+}\right]_{3}\right)=d-d=0, & n=3, \\
\frac{1}{2}\left(\left[\left[\mathrm{JG}^{+}\right]_{1} \mathrm{G}^{-}\right]_{n}+\left[\left[\mathrm{JG}^{-}\right]_{1} \mathrm{G}^{+}\right]_{n}\right)=0, & n>3 .\end{cases}
\end{aligned}
$$

(3) Next we show that $\mathrm{G}^{+}(z) \cdot \mathrm{F}(w)=0$. Observe that

$$
\left[\mathrm{G}^{+} \mathrm{F}\right]_{n}=\left[\mathrm{G}^{+}\left[\mathrm{G}^{+} \Phi\right]_{1}\right]_{n}=-\left[\mathrm{G}^{+}\left[\mathrm{G}^{+} \Phi\right]_{n}\right]_{1} .
$$

The right-hand side vanishes for $n>1$, while it equals the negative of the left-hand side for $n=1$. 
(4) We next calculate $\mathrm{G}^{-}(z) \cdot \mathrm{T}(w)$. We will use the fact that

$$
\left[\mathrm{G}^{ \pm}(\partial \mathrm{J})\right]_{n}=\partial\left[\mathrm{G}^{ \pm} \mathrm{J}\right]_{n}+\left[\left(\partial \mathrm{G}^{ \pm}\right) \mathrm{J}\right]_{n}= \begin{cases}\mp \partial \mathrm{G}^{ \pm}, & n=1 \\ \pm \mathrm{G}^{ \pm}, & n=2, \\ 0, & n>2 .\end{cases}
$$

Also, observe that

$$
\left[\mathrm{G}^{-}\left[\mathrm{G}^{-} \mathrm{G}^{+}\right]_{1}\right]_{n}=\left[\left[\mathrm{G}^{-} \mathrm{G}^{-}\right]_{1} \mathrm{G}^{+}\right]_{n}-\left[\mathrm{G}^{-}\left[\mathrm{G}^{-} \mathrm{G}^{+}\right]_{n}\right]_{1} \text {. }
$$

For $n=1$, this shows that $\left[\mathrm{G}^{-}\left[\mathrm{G}^{-} \mathrm{G}^{+}\right]_{1}\right]_{1}=\frac{1}{2}\left[\mathrm{FG}^{+}\right]_{1}=0$, and thus that

$$
\left[\mathrm{G}^{-} \mathrm{T}\right]_{n}=\left[\mathrm{G}^{-}\left[\mathrm{G}^{-} \mathrm{G}^{+}\right]_{1}\right]_{n}+\frac{1}{2}\left[\mathrm{G}^{-}(\partial \mathrm{J})\right]_{n}=\frac{1}{2} \partial \mathrm{G}^{-} \text {. }
$$

For $n=2$, we see that

$$
\left[\mathrm{G}^{-} \mathrm{T}\right]_{2}=\left[\mathrm{G}^{-}\left[\mathrm{G}^{-} \mathrm{G}^{+}\right]_{1}\right]_{2}+\frac{1}{2}\left[\mathrm{G}^{-}(\partial \mathrm{J})\right]_{2}=-\left[\mathrm{G}^{-}\left[\mathrm{G}^{-} \mathrm{G}^{+}\right]_{2}\right]_{1}-\frac{1}{2} \mathrm{G}^{-}=-\frac{3}{2} \mathrm{G}^{-} .
$$

Similar reasoning shows that $\left[\mathrm{G}^{-} \mathrm{T}\right]_{n}=0$ for $n>2$.

The calculation of $\mathrm{G}^{+}(z) \cdot \mathrm{T}(w)$ is similar, except that we use the formula $\mathrm{T}=\left[\mathrm{G}^{+} \mathrm{G}^{-}\right]_{1}-$ $\frac{1}{2} \partial \mathrm{J}$.

(5) Next, we calculate $\mathrm{T}(z) \cdot \mathrm{T}(w)$ :

$$
\begin{aligned}
{[\mathrm{TT}]_{n}=} & \frac{1}{2}\left(\left[\mathrm{~T}\left[\mathrm{G}^{+} \mathrm{G}^{-}\right]_{1}\right]_{n}+\left[\mathrm{T}\left[\mathrm{G}^{-} \mathrm{G}^{+}\right]_{1}\right]_{n}\right) \\
= & \frac{1}{2}\left((n-1)\left[\left[\mathrm{TG}^{+}\right]_{2} \mathrm{G}^{-}\right]_{n-1}+\left[\left[\mathrm{TG}^{+}\right]_{1} \mathrm{G}^{-}\right]_{n}\right. \\
& \left.+(n-1)\left[\left[\mathrm{TG}^{-}\right]_{2} \mathrm{G}^{+}\right]_{n-1}+\left[\left[\mathrm{TG}^{-}\right]_{1} \mathrm{G}^{+}\right]_{n}\right) \\
& +\frac{1}{2}\left(\left[\mathrm{G}^{+}\left[\mathrm{TG}^{-}\right]_{n}\right]_{1}+\left[\mathrm{G}^{-}\left[\mathrm{TG}^{+}\right]_{n}\right]_{1}\right) .
\end{aligned}
$$

For $n=1$, this equals

$$
\begin{aligned}
\frac{1}{2}\left(\left[\left(\partial \mathbf{G}^{+}\right) \mathbf{G}^{-}\right]_{1}+\left[\left(\partial \mathbf{G}^{-}\right) \mathbf{G}^{+}\right]_{1}+\left[\mathrm{G}^{+}\left(\partial \mathbf{G}^{-}\right)\right]_{1}+\left[\mathbf{G}^{-}\left(\partial \mathbf{G}^{+}\right)\right]_{1}\right) & \\
& =\frac{1}{2}\left(\partial\left[\mathrm{G}^{+} \mathbf{G}^{-}\right]_{1}+\partial\left[\mathrm{G}^{-} \mathbf{G}^{+}\right]_{1}\right)=\partial \mathrm{T} .
\end{aligned}
$$

For $n=2$, we obtain

$$
\begin{aligned}
\frac{1}{2}\left(\left[\left[\mathrm{TG}^{+}\right]_{2} \mathrm{G}^{-}\right]_{1}+\left[\left[\mathrm{TG}^{-}\right]_{2} \mathrm{G}^{+}\right]_{1}+\left[\left[\mathrm{TG}^{+}\right]_{1} \mathrm{G}^{-}\right]_{2}+\right. & {\left[\left[\mathrm{TG}^{-}\right]_{1} \mathrm{G}^{+}\right]_{2} } \\
& \left.+\left[\mathrm{G}^{+}\left[\mathrm{TG}^{-}\right]_{2}\right]_{1}+\left[\mathrm{G}^{-}\left[\mathrm{TG}^{+}\right]_{2}\right]_{1}\right)=2 \mathrm{~T} .
\end{aligned}
$$

For $n=3$, we obtain

$$
\frac{1}{2}\left(2\left[\left[\mathrm{TG}^{+}\right]_{2} \mathrm{G}^{-}\right]_{2}+2\left[\left[\mathrm{TG}^{-}\right]_{2} \mathrm{G}^{+}\right]_{2}+\left[\left[\mathrm{TG}^{+}\right]_{1} \mathrm{G}^{-}\right]_{3}+\left[\left[\mathrm{TG}^{-}\right]_{1} \mathrm{G}^{+}\right]_{3}\right)=0 .
$$

Finally, for $n=4$ we obtain

$$
\frac{1}{2}\left(3\left[\left[\mathrm{TG}^{+}\right]_{2} \mathrm{G}^{-}\right]_{3}+3\left[\left[\mathrm{TG}^{-}\right]_{2} \mathrm{G}^{+}\right]_{2}+\left[\left[\mathrm{TG}^{+}\right]_{1} \mathrm{G}^{-}\right]_{4}+\left[\left[\mathrm{TG}^{-}\right]_{1} \mathrm{G}^{+}\right]_{4}\right)=\frac{3}{2} d .
$$

(6) From the vanishing of $\mathrm{G}^{-}(z) \cdot \Phi(w)$, it follows that $[\Phi \mathrm{F}]_{n}=-\frac{1}{2}\left[\Phi\left[\mathrm{G}^{-} \mathrm{G}^{-}\right]_{1}\right]_{n}=0$ for all $n>0$. From this, we see that $[\mathrm{FF}]_{n}=\left[\mathrm{G}^{+}[\Phi \mathrm{F}]_{n}\right]_{1}=0$. 
(7) We now calculate $\mathrm{G}^{-}(z) \cdot \mathrm{F}(w)$. Using the fact $[\mathrm{J} \Phi]_{1}=-3 \Phi$, we see that

$$
3 \Phi=[\Phi \mathrm{J}]_{1}=-\left[\Phi\left[\mathrm{G}^{-} \mathrm{G}^{+}\right]_{2}\right]_{1}=\left[\mathrm{G}^{-}\left[\Phi \mathrm{G}^{+}\right]_{1}\right]_{2}=\left[\mathrm{G}^{-} \mathrm{F}\right]_{2} .
$$

Incidentally, this shows that

$$
[\Phi \Phi]_{n}=\frac{1}{3}\left[\Phi\left[\mathrm{G}^{-} \mathrm{F}\right]_{2}\right]_{n}=-\frac{1}{3}\left[\mathrm{G}^{-}[\Phi \mathrm{F}]_{n}\right]_{2}=0 .
$$

A similar calculation shows that $\left[\mathrm{G}^{-} \mathrm{F}\right]_{n}=0$ for $n>2$. Since

$$
\left[\mathrm{G}^{-} \mathrm{F}\right]_{1}=-\frac{1}{2}\left[\mathrm{G}^{-}\left[\mathrm{G}^{-} \mathrm{G}^{-}\right]_{1}\right]_{1}=-\frac{1}{2}\left[\left[\mathrm{G}^{-} \mathrm{G}^{-}\right]_{1} \mathrm{G}^{-}\right]_{1}+\frac{1}{2}\left[\mathrm{G}^{-}\left[\mathrm{G}^{-} \mathrm{G}^{-}\right]_{1}\right]_{1},
$$

we see that $2\left[\mathrm{G}^{-} \mathrm{F}\right]_{1}=\left[\mathrm{FG}^{-}\right]_{1}$. But $\left[\mathrm{FG}^{-}\right]_{1}+\left[\mathrm{G}^{-} \mathrm{F}\right]_{1}=\partial\left[\mathrm{G}^{-} \mathrm{F}\right]_{2}=3 \partial \Phi$, and we conclude that $\left[\mathrm{G}^{-} \mathrm{F}\right]_{1}=\partial \Phi$.

(8) We now calculate $\Phi(z) \cdot \mathrm{T}(w)$ :

$$
[\Phi \mathrm{T}]_{n}=\frac{1}{2}\left(\left[\Phi\left[\mathrm{G}^{+} \mathrm{G}^{-}\right]_{1}\right]_{n}+\left[\Phi\left[\mathrm{G}^{-} \mathrm{G}^{+}\right]_{1}\right]_{n}\right)= \begin{cases}\frac{1}{2}\left(\left[\mathrm{FG}^{-}\right]_{1}-\left[\mathrm{G}^{-} \mathrm{F}\right]_{1}\right)=\frac{1}{2} \partial \Phi, & n=1, \\ \frac{1}{2}\left[\mathrm{FG}^{-}\right]_{2}=\frac{3}{2} \mathrm{~F}, & n=2, \\ \frac{1}{2}\left[\mathrm{FG}^{-}\right]_{n}=0, & n>2 .\end{cases}
$$

The formula for $\mathrm{T}(z) \cdot \mathrm{F}(w)$ is easily obtained from this, since $\mathrm{F}=\left[\mathrm{G}^{+} \Phi\right]_{1}$.

\section{REFERENCES}

1. O. Aharony, O. Ganor, N. Sochen, J. Sonnenschein and S. Yankielowicz, Physical states in G/G models and two-dimensional gravity, Nucl. Phys. B399 (1993), 527-559.

2. O. Aharony, O. Ganor, J. Sonnenschein and S. Yankielowicz, On the twisted $G / H$ topological models, Nucl. Phys. B399 (1993), 560-580.

3. A.A. Belavin, A.M. Polyakov and A. M. Zamolodchikov, Infinite conformal symmetry in twodimensional quantum field theories, Nucl. Phys. B241 (1984), 333-380.

4. R. E. Borcherds, Vertex operator algebras, Kac-Moody algebras and the monster, Proc. Natl. Acad. Sci. USA 83 (1986), 3068-3071.

5. V.G. Drinfeld, Quasi-Hopf algebras, Leningrad Math. J. $11419-1457$.

6. J.M. Figueroa-O'Farill, Affine algebras, $N=2$ superconformal algebras, and gauged WZNW models, hep-th/9306164.

7. E. Frenkel, Cohomology of Kazama-Suzuki model, Appendix to [16].

8. E.Getzler, Batalin-Vilkovisky algebras and two-dimensional topological field theories, hepth/9212043, to appear, Commun. Math. Phys.

9. E. Getzler, Two-dimensional topological gravity and equivariant cohomology, hep-th/9305013, to appear, Commun. Math. Phys.

10. Hong-Liang $\mathrm{Hu}$ and Ming $\mathrm{Yu}$, On the equivalence of non-critical strings and $G_{k} / G_{k}$ topological field theories, Phys. Lett. B 289 (1992), 302-308.

11. J.M. Isidro and A.V. Ramallo, Topological current algebras in two dimensions, hep-th/9307176.

12. V.G. Kac and I.T. Todorov, Superconformal current algebras and their unitary representations, Commun. Math. Phys. 102 (1985), 337-347.

13. D. Karabali and H.J. Schnitzer, BRST quantization of the gauged WZW action and coset conformal field theories, Nucl. Phys. B329 (1990), 649-666.

14. Y. Kazama, Novel topological field theories, Mod. Phys. Lett. A6 (1991), 1321-1332. 
15. Y. Kazama and H. Suzuki, New $N=2$ superconformal field theories and superstring compactification, Nucl. Phys. B321 (1989), 232-268.

16. S. Mukhi and C. Vafa, Two dimensional black-hole as a topological coset model of $c=1$ string theory, hep-th/9301183.

17. S. Parkhomenko, Extended superconformal current algebras and finite-dimensional Manin triples, Sov. Phys. JETP, 75 (1992), 1-3.

18. A. Schwarz and M. Penkava, On some algebraic structures arising in string theory, hep-th/912071.

19. H.J. Schnitzer, A path integral construction of superconformal field theories from a gauged supersymmetric Wess-Zumino-Witten action, Nucl. Phys. B324 (1989), 412-426.

20. M.A. Semenov-Tian-Shansky, What a classical r-matrix is, Functional Anal. Appl. 17 (1983), 259272 .

21. A. Sevrin, W. Troust, A. van Proyen and Ph. Spindel, Extended supersymmetric $\sigma$-models on group manifolds (II). Current algebras. Nucl. Phys. B211 (1988/89), 465-492.

22. Ph. Spindel, A. Sevrin, W. Troust and A. van Proyen, Extended supersymmetric $\sigma$-models on group manifolds (I). The complex structures. Nucl. Phys. B308 (1988), 662-698.

23. K. Thielemans, A Mathematica package for computing operator product expansions, Int. Jour. Mod. Physics C2 (1991), 787-798.

Department of Mathematics, Mit, Cambridge MA 02139 USA

E-mail address: getzler@math.mit.edu 